\title{
UNIQUE EXTREMALITY
}

\author{
By \\ V. BoŽIN, N. LAKIC, V. MARKOVIĆ AND M. MATELJEVIĆ
}

\section{Introduction}

Extremal mappings have been one of the main topics in the theory of quasiconformal mappings since its earliest days, when Grötzsch solved the extremal problem for two rectangles. Grötzsch showed that among all quasiconformal mappings from a rectangle $R_{1}$ onto another rectangle $R_{2}$, mapping the sides of $R_{1}$ onto the corresponding sides of $R_{2}$, there exists a unique mapping with minimal dilatation (see [Gr]). Later, Teichmüller [T] generalized Grötzsch's ideas and found many extremal quasiconformal mappings, including the uniquely extremal mapping $f$ in the set of all quasiconformal mappings homotopic to a given sense-preserving homeomorphism between two compact Riemann surfaces $R$ and $S=f(R)$ of genus greater than 1 . In a neighborhood of all but finitely many points on the compact Riemann surface $R$, the extremal map $f$ could be expressed as a conformal map followed by an affine map followed by a conformal map. The Beltrami coefficient of $f$ is of the form $k|\varphi| / \varphi$, where $0 \leq k<1$ and $\varphi$ is an integrable holomorphic quadratic differential.

The modern Teichmüller theory began in the 1950s with Ahlfors and Bers. They considered the space QC $(R)$ of all quasiconformal mappings $f$ from a given Riemann surface $R$ to a variable Riemann surface $f(R)$. The Teichmüller space $T(R)$ is the space of these mappings factored by an equivalence relation. Two mappings, $f$ and $g$, are equivalent if there is a conformal mapping $c$ from $f(R)$ onto $g(R)$ and a homotopy through quasiconformal mappings $h_{t}$ mapping $R$ onto $g(R)$ such that $h_{0}=c \circ f, h_{1}=g$ and $h_{t}(p)=c \circ f(p)=g(p)$ for every $p$ in the ideal boundary of $R$. We denote the equivalence class of a quasiconformal mapping $f$ in $\mathrm{QC}(R)$ by $[f]$ or $[\mu]$, where $\mu$ is the Beltrami coefficient of $f$. Thus, the Teichmüller space $T(R)$ may also be represented as the space of equivalence classes of Beltrami coefficients $\mu$ in the unit ball $M(R)$ of the space $L^{\infty}(R)$ of all essentially bounded

\footnotetext{
${ }^{*}$ Research of the second author supported in part by NSF grant DMS 9706769.
} 
Beltrami differentials on $R$. When a Riemann surface $R$ is hyperbolic, two Beltrami differentials in $M(R)$ are equivalent if they induce quasiconformal mappings on $R$ whose lifts to the unit disc $\Delta$ have extensions to the closure of $\Delta$ with the same boundary values. The equivalence class of the zero differential is the basepoint in $T(R)$. If $\tau$ is in $T(R)$, we let $k_{0}(\tau)$ be the infimum of $k(\mu)=\|\mu\|_{\infty}$ over all Beltrami differentials $\mu$ in $M(R)$ with $[\mu]=\tau$. We define

$$
K(\mu)=\frac{1+k(\mu)}{1-k(\mu)}
$$

and

$$
K_{0}([\mu])=\frac{1+k_{0}([\mu])}{1-k_{0}([\mu])} .
$$

The Teichmüller distance between two points $\tau_{1}=\left[\mu_{1}\right]$ and $\tau_{2}=\left[\mu_{2}\right]$ in $T(R)$ is $\frac{1}{2} \ln K_{0}([\mu])$, where $\mu$ is the Beltrami coefficient of $f^{\mu_{1}} \circ\left(f^{\mu_{2}}\right)^{-1}$. Here $f^{\mu_{1}}$ and $f^{\mu_{2}}$ are quasiconformal mappings with domain $R$ and Beltrami coefficients $\mu_{1}$ and $\mu_{2}$, respectively. If $k(\mu)=k_{0}([\mu])$, we say that $\mu$ is an extremal Beltrami coefficient in its Teichmüller class. $\mu$ is uniquely extremal in its Teichmüller class if every other Beltrami coefficient $\nu$ in the same class satisfies $\|\nu\|_{\infty}>\|\mu\|_{\infty}$. If a Beltrami coefficient $\mu$ is of the form $k|\varphi| / \varphi$, with $0 \leq k<1$ and $\varphi$ an integrable holomorphic quadratic differential, then $\mu$ is uniquely extremal in its Teichmüller class (see [S4]). Every Beltrami coefficient $\mu$ in $M(R)$ generates a complex structure on $R$, and the Teichmüller space $T(R)$ is the space of deformations of a given (basepoint) complex structure on a Riemann surface $R$. The Teichmüller metric is natural; it measures the distance between different conformal structures.

If a Riemann surface $R$ is of finite analytic type, that is, conformally equivalent to a compact Riemann surface with finitely many points removed, the Teichmüller space $T(R)$ is finite-dimensional, and every point $\tau$ in $T(R)$ is represented by a uniquely extremal Beltrami coefficient $\mu$. When $T(R)$ is of infinite analytic type, the situation is more difficult and all three cases may occur: a Beltrami coefficient $\mu \in M(R)$ may be uniquely extremal, extremal but not uniquely extremal, or nonextremal. Not all extremal quasiconformal mappings are of the form $k|\varphi| / \varphi$, with $0 \leq k<1$ and $\varphi$ an integrable holomorphic quadratic differential. In [BA] Beurling and Ahlfors showed

$$
z \mapsto z|z|^{K-1}
$$

is extremal among all quasiconformal homeomorphisms of the upper half plane $H$ with the same boundary values on the real axis. If $w=h(z)=\frac{1}{2} \ln (z)-\frac{1}{4} \pi$ is a conformal mapping from the upper half plane to the horizontal strip $S=$ $\left\{w:|w-\bar{w}|<\frac{1}{2} \pi\right\}$ then $h f h^{-1}$ is a horizontal stretch in $S$. Strebel proved in [S3] 
that this stretching is uniquely extremal in its class in $T(S)$; thus $f$ is uniquely extremal in its class in $T(H)$.

In [S4], Strebel made another breakthrough by constructing the first example of a nonuniquely extremal Beltrami coefficient. Strebel considered the plane region

$$
V=\{z=x+i y:|x|<1\} \cup\{z: y<0\}
$$

now known as Strebel's chimney. For every real number $K>1$, the quasiconformal homeomorphism $f_{K}(z)=x+i K y$ of $V$ is extremal in its class $\tau_{K} \in T(V)$. On the other hand, $\tau_{K}$ contains infinitely many distinct extremal mappings. For instance, the function $h_{L}(z)=f_{K}(z)$ for $y \geq 0$ and $h_{L}(z)=f_{L}(z)$ for $y<0$ is extremal in $\tau_{K}$ for every $L \in[1 / K, K]$.

Ahlfors and Bers showed $T(R)$ has a complex structure with tangent space at the basepoint isomorphic to Banach space $A(R)^{*}$, where $A(R)$ is the space of integrable quadratic differentials $\varphi$ holomorphic on $R$. The complex structure on $T(R)$ comes from the complex structure of the unit ball $M(R)$ in the complex vector space of $L^{\infty}$ complex valued Beltrami differentials on $R$. Two tangent vectors $\mu$ and $\nu$ in the tangent space to $M(R)$ determine the same tangent vector in $T(R)$ if, and only if,

$$
\int_{R} \varphi \mu=\int_{R} \varphi \nu \quad \text { for all } \varphi \in A(R) .
$$

If $\mu$ and $\nu$ have this property, we say that they represent the same Teichmüller infinitesimal equivalence class or, more briefly, that they are infinitesimally equivalent. The space of equivalence classes is denoted by $B(R)$. A given $\mu$ is said to be extremal in its infinitesimal Teichmüller class if $\|\mu\|_{\infty} \leq\|\nu\|_{\infty}$ for any $\nu$ infinitesimally equivalent to $\mu$. It is uniquely extremal in its infinitesimal class if $\|\mu\|_{\infty}<\|\nu\|_{\infty}$ for every other $\nu$ infinitesimally equivalent to $\mu$. Equivalently, an extremal Beltrami differential $\mu \in L^{\infty}(R)$ is uniquely extremal in its infinitesimal Teichmüller class if the linear functional $\Lambda_{\mu} \in A(R)^{*}$ induced by $\mu, \Lambda_{\mu}(\varphi)=\int_{R} \varphi \mu$, has a unique norm-preserving extension to a bounded linear functional on $L^{1}(R)$. Since the Riemann surface $R$ will be fixed in our discussion, we denote the Teichmüller space $T(R)$ by $T$ and the tangent space $B(R)$ by $B$. Every Beltrami differential $\mu$ in $M(R)$ defines two equivalence classes, one in $T$ and another in $B$. We denote both of them by $[\mu]$, whenever there is no ambiguity. In particular, $\mu$ is extremal in its class (in $T$ or $B$ ) if and only if $\|\nu\|_{\infty} \geq\|\mu\|_{\infty}$ for every $\nu$ equivalent to $\mu$.

Hamilton, Krushkal, Reich and Strebel ([H], [K], [RS]) showed that a Beltrami coefficient $\nu$ in $M(R)$ is extremal in its class in $T$ if and only if $\nu$ is extremal in its class in $B$. It was natural to consider whether the analogous statement holds for 
the unique extremality. In several articles Reich and Strebel showed that in many special situations the two notions of unique extremality coincide and conjectured that the notions may coincide in general. In this paper we prove the answer to this conjecture is affirmative.

Equivalence Theorem A Beltrami coefficient $\mu$ in $M(R)$ is uniquely extremal in its Teichmüller class if, and only if, it is uniquely extremal in its infinitesimal Teichmüller class.

To prove this theorem, we need estimates which allow us to compare two Beltrami coefficients $\mu$ and $\nu$ in the same global equivalence class and two Beltrami differentials in the same infinitesimal equivalence class. These estimates generalize Reich's $\delta$-inequality for Beltrami differentials in the same equivalence class (see [R2]). Unlike Reich's forms of the $\delta$-inequalities, our forms do not require either of the Beltrami coefficients to have constant absolute value.

Since the conditions we give on an infinitesimal class are linear, we conclude that certain properties of $\mu$ are invariant under multiplication by $t$, for $|t|<1 /\|\mu\|_{\infty}$. In particular, we show that if $\mu$ is uniquely extremal, then so is $t \mu$; and we give additional necessary and sufficient criteria for the uniqueness of a geodesic joining a pair of points in Teichmüller space. We also show that holomorphic motions generated by uniquely extremal Beltrami coefficients have unique Slodkowski extensions.

The generalized $\delta$-inequality is our first step towards obtaining the criterion for the unique extremality of Beltrami differentials. The next important step is the analysis of the proof of the Hahn-Banach theorem and its applications to our setting. We obtain four necessary and sufficient criteria for the unique extremality of a given Beltrami coefficient $\mu$.

The first criterion says that for every admissible variation $\eta$ of $\mu$ and every compact subset $E$ of the extremal set $X(\eta)$ of $\eta$ with positive measure, there exists a sequence of unit vectors $\varphi_{n} \in A(R)$ such that

$$
\frac{1}{\int_{E}\left|\varphi_{n}\right|}\left(\|\eta\|_{\infty}-\operatorname{Re} \int_{R} \eta \varphi_{n}\right) \rightarrow 0 .
$$

Here, an admissible variation $\eta$ of $\mu$ is any Beltrami differential which does not increase the $L^{\infty}$-norm of $\mu$, and which is allowed to differ from $\mu$ only on the set where $|\mu(z)| \leq$ constant $<\|\mu\|_{\infty}$; and the extremal set $X(\eta)$ is the set where $|\eta(z)|=\|\eta\|_{\infty}$. This criterion is analogous to the Hamilton-Krushkal, ReichStrebel necessary and sufficient criterion for extremality. Namely, $\mu$ is extremal if, and only if, there is a sequence $\varphi_{n}$ of holomorphic quadratic differentials of norm 
1 such that

$$
\|\mu\|_{\infty}-\operatorname{Re} \int_{R} \mu \varphi_{n} \rightarrow 0 .
$$

The second criterion says that an extremal Beltrami coefficient $\mu$ is uniquely extremal in its Teichmüller class if, for every admissible variation $\eta$ of $\mu$, the truncation

$$
\left[\eta \chi_{E}+\frac{1}{1+r} \eta \chi_{R-E}\right] \quad(\text { where } r>0)
$$

of the complex structure outside any compact subset $E$ of $X(\eta)$ represents a Strebel point.

The third criterion says that that the linear functional $\lambda_{\mu}(\varphi)=\operatorname{Re} \int_{R} \mu \varphi$ satisfies the unique approximation property at each $x_{0} \in L^{1}(R)-A(R)$, that is, there exist sequences $u_{n}$ and $v_{n}$ in $A(R)$, such that

$$
\|\lambda\|\left(\left\|u_{n}-x_{0}\right\|+\left\|x_{0}-v_{n}\right\|\right)-\lambda\left(u_{n}-v_{n}\right) \rightarrow 0
$$

The fourth criterion says that for every admissible variation $\eta$ of $\mu$, there exists a sequence $\varphi_{n}$ in $A(R)$ such that

(i) $\delta\left(\varphi_{n}\right)=\left\|\varphi_{n}\right\|\|\eta\|_{\infty}-\operatorname{Re} \int_{R} \varphi_{n} \eta \rightarrow 0$, and

(ii) $\liminf _{n \rightarrow 0}\left|\varphi_{n}(z)\right|>0$ for almost all $z$ in $X(\eta)$.

In [R1] and [R4], Reich showed that the fourth criterion is sufficient in several special cases, and so we call this criterion Reich's criterion. This criterion is another analogue of the Hamilton-Krushkal, Reich-Strebel criterion for the extremality.

These four criteria are among those listed in the theorem in Section 11, which we call the Characterization Theorem. The Characterization Theorem applies to many interesting situations. For instance, we can say precisely when a Beltrami differential of the form $k|\varphi(z)| / \varphi(z)$, with $\varphi$ a holomorphic quadratic differential with $\|\varphi\|=\infty$, is uniquely extremal.

Theorem. Let $\varphi$ be a nonzero holomorphic quadratic differential on a Riemann surface $R$, and let $f$ be a quasiconformal mapping with domain $R$ and Beltrami coefficient $\mu=k|\varphi| / \varphi, 0<k<1$. Then $f$ is uniquely extremal if and only if there exists a sequence $\varphi_{n}$ in $A(R)$ such that

(1) $\varphi_{n}(z)$ converges to $\varphi(z)$ uniformly on compact subsets of $R$, and

(2) $k\left\|\varphi_{n}\right\|-\operatorname{Re} \int_{R} \varphi_{n} \mu \rightarrow 0$.

There are many examples of extremal Beltrami differentials with non-constant modulus (see Section 2 or [EL] and [L1] for more details), but all examples of uniquely extremal Beltrami differentials known up to now were of the general Teichmüller type $k|\varphi(z)| / \varphi(z)$, where $0<k<1$ and $\varphi$ is a suitably chosen 
quadratic differential on $R$. Moreover, many results obtained studying the extremal problems speak in favour of the conjecture that all uniquely extremal Beltrami differentials $\mu$ satisfy $|\mu(z)|=\|\mu\|_{\infty}$ for almost all $z$. Suprisingly, we disprove this conjecture and show that there are uniquely extremal Beltrami differentials with non-constant modulus on any Riemann surface which is a subsurface of another Riemann surface and for which the complement contains a cluster point. Thus, our counterexample applies to all Riemann surfaces of finite genus and infinite analytic type and to all surfaces covered by Fuchsian groups of the second kind. The construction of the counterexample shows that we can start with an arbitrary Beltrami coefficient $\mu$, and obtain a uniquely extremal Beltrami coefficient $\nu$ such that $\nu$ differs from $\mu$ only on the set of arbitrary small positive measure.

Theorem. Let $R$ be a Riemann surface which is a subset of another Riemann surface $W$ such that $W-R$ has a cluster point, and let $q$ be a unit vector in $A(R)$. Then for every $\epsilon>0$ and every Beltrami differential $\mu$ in $M(R)$, there exists a uniquely extremal Beltrami coefficient $\nu$ on $R$ such that the q-measure of the set where $\mu$ is different from $\nu$ is less than $\epsilon$.

Our first example of a uniquely extremal Beltrami coefficient with non-constant modulus vanishes on some Mergelyan set $M$ with positive measure, that is, on a compact set with positive measure, empty interior and connected complement. Outside $M, \mu$ is equal to the limit of $\left|\varphi_{n}\right| / \varphi_{n}$ for a sequence of quadratic differentials $\varphi_{n}$ in $A(R)$, carefully chosen so that $\mu$ satisfies Reich's condition on $R-M$. Using the Characterization Theorem, we can strengthen the construction of the counterexample and obtain an example of uniquely extremal Beltrami differential $\mu$ for which $|\mu(z)|$ is never equal to $\|\mu\|_{\infty}$, that is, $X(\mu)=\emptyset$.

\section{The boundary dilatation}

The boundary dilatation $H([\mu])$ of the Teichmüller class of $\mu$ is the infimum over all elements $\nu$ in the equivalence class of $\mu$ in $T$ of the quantity $\left(1+h^{*}(\nu)\right) /\left(1-h^{*}(\nu)\right)$. Here $h^{*}(\nu)$ is the infimum over all compact subsets $F$ contained in $R$ of the essential supremum of the Beltrami differential $\nu(z)$ as $z$ varies over $R-F$. As usual, we let

$$
H^{*}(\mu)=\frac{1+h^{*}(\mu)}{1-h^{*}(\mu)}
$$

and

$$
h([\mu])=\frac{H([\mu])-1}{1+H([\mu])} .
$$


The inequalities $H^{*}(\mu) \leq K(\mu)$ and $H(\tau) \leq K_{0}(\tau)$ are obvious. We call $\tau$ in $T(R)$ a Strebel point if $H(\tau)<K_{0}(\tau)$ (see [EL] and [L1]). Clearly, the basepoint is not a Strebel point, since $H([0])=K_{0}([0])=1$. By Strebel's frame mapping theorem, every Strebel point $\tau$ is represented by the unique Beltrami differential of the form $k|\varphi| / \varphi$, where $k=k_{0}(\tau) \in(0,1)$ and $\varphi$ is a unit vector in $A(R)$. Strebel points play a central role in our investigation, as they have in previous results in Teichmüller geometry (see [EL] and [L1]).

In a parallel manner we can define the boundary semi-norm $b$ on the infinitesimal level. The boundary semi-norm $b([\mu])$ of the infinitesimal Teichmüller class of $\mu$ is the infimum over all elements $\nu$ in the equivalence class of $\mu$ in $B$ of the quantity $b^{*}(\nu)$. Here $b^{*}(\nu)$ is the infimum over all compact subsets $F$ contained in $R$ of the essential supremum of the Beltrami differential $\nu(z)$ as $z$ varies over $R-F$. An infinitesimal version of Strebel's frame mapping theorem is the following:

Theorem (Infinitesimal frame mapping theorem). If $v$ is in $B$ and $b(v)<\|v\|$, then there exists a unique quadratic differential $\varphi$ in $A(R)$ of norm equal to one, such that $\|v\||\varphi| / \varphi$ represents $v$.

The proof of Strebel's frame mapping condition can be found in [S2] and [G], and the proof of its infinitesimal version is in Theorem 2.4 in [R5] (see also [EG] and [L2]).

\section{The main inequalities}

Let $f$ be any quasiconformal mapping with domain $R$, and let $\mu$ be its Beltrami coefficient.

Suppose first that $\nu$ is a Beltrami coefficient in $M(R)$ such that $\mu$ and $\nu$ belong to the same class in $T$. Then there exists a quasiconformal mapping $g$ with domain $R$ and Beltrami coefficient $\nu$ such that $f$ and $g$ coincide on the ideal boundary of $R$. Let $\tilde{\mu}$ be the Beltrami coefficient of $f^{-1}$ and let $\tilde{\nu}$ be the Beltrami coefficient of $g^{-1}$. If $\varphi$ is any quadratic differential in $A(R)$, then, by studying the trajectory structure of $\varphi$, Reich and Strebel showed

$$
\|\varphi\| \leq \int_{R}|\varphi| \frac{\left|1-\xi \frac{\varphi}{|\varphi|}\right|^{2}}{1-|\xi|^{2}}
$$

where $\xi$ is the Beltrami differential of the composition $g^{-1} \circ f$. A simple calculation 
shows that this inequality is equivalent to the following Main Inequality:

$$
|\varphi|\left|\leq \int_{R}\right| \varphi \mid \frac{\left|1-\mu \frac{\varphi}{|\varphi|}\right|^{2}}{1-|\mu|^{2}} \frac{\left|1+\mu \frac{\tilde{\nu}(f)}{\tilde{\mu}(f)} \frac{\varphi}{|\varphi|} \frac{1-\bar{\mu} \frac{\bar{\varphi}}{|\varphi|}}{1-\mu \frac{\varphi}{|\varphi|}}\right|^{2}}{1-|\tilde{\nu}(f)|^{2}} .
$$

There are two consequences of the Main Inequality, known as the fundamental Reich-Strebel inequalities. For the proofs, see [G]. If $K_{0}=K_{0}([\mu])$, then

$$
\frac{1}{K_{0}}\|\varphi\| \leq \int|\varphi| \frac{\left|1-\mu \frac{\varphi}{|\varphi|}\right|^{2}}{1-|\mu|^{2}}
$$

and, if there exists a quadratic differential $\varphi_{0}$ in $A(X)$ such that $\left\|\varphi_{0}\right\|=1$, and $[\mu]=\left[k\left|\varphi_{0}\right| / \varphi_{0}\right]$ in $T$ for some $k \in[0,1)$, then

$$
\frac{1+k}{1-k}=K_{0} \leq \int\left|\varphi_{0}\right| \frac{\left|1+\mu \frac{\varphi_{0}}{\left|\varphi_{0}\right|}\right|^{2}}{1-|\mu|^{2}} .
$$

Suppose now that $\nu$ is a Beltrami differential in $M(R)$ such that $\mu$ and $\nu$ belong to the same class in $B$. Then $\int_{R} \mu \varphi=\int_{R} \nu \varphi$ for all $\varphi$ in $A(R)$. That yields

$$
\begin{aligned}
\operatorname{Re} \int_{R} \frac{(1-\bar{\nu} \mu)(\mu-\nu)}{1-|\nu|^{2}} \varphi & =\operatorname{Re} \int_{R}\left(\frac{(1-\bar{\nu} \mu)(\mu-\nu)}{1-|\nu|^{2}}-(\mu-\nu)\right) \varphi \\
& =-\operatorname{Re} \int_{R} \frac{(\mu-\nu)^{2} \bar{\nu}}{1-|\nu|^{2}} \varphi \leq \int_{R} \frac{|\mu-\nu|^{2}}{1-|\nu|^{2}}|\varphi|
\end{aligned}
$$

Therefore,

$$
\operatorname{Re} \int_{R} \frac{(1-\bar{\nu} \mu)(\mu-\nu)}{1-|\nu|^{2}} \varphi \leq \int_{R} \frac{|\mu-\nu|^{2}}{1-|\nu|^{2}}|\varphi| .
$$

Combining the integrands of this inequality we obtain

$$
\int_{R}|\varphi|\left(1-|\mu|^{2}\right) \leq \int_{R}|\varphi|\left|1-\mu \frac{\varphi}{|\varphi|}\right|^{2} \frac{1+\left.\nu \frac{\varphi}{|\varphi|} \frac{1-\left.\bar{\mu} \frac{\bar{\varphi}}{|\varphi|}\right|^{2}}{1-\mu \frac{\varphi}{|\varphi|}}\right|^{2} .|\nu|^{2}}{1-}
$$

We call inequality (5) the Infinitesimal Main Inequality.

For the proof of the Main Inequality, see, for example, [G]; and, for more details on the calculation used to prove the Infinitesimal Main Inequality, see [R1]. 


\section{The proof of the Equivalence Theorem when $\mu$ has constant absolute value}

In this section we assume that $\mu$ in $B(R)$ has constant absolute value and $\|\mu\|_{\infty}=k<\infty$. We first prove

Lemma 1. If $\mu$ has constant absolute value and is uniquely extremal in its class in $T$, then $\mu$ is uniquely extremal in its class in $B$.

Proof. Let $\mu$ be uniquely extremal in its Teichmüller class with $|\mu(z)| \equiv k$. Suppose that $\mu$ is not uniquely extremal in its infinitesimal class. Then there exists a Beltrami differential $\nu$ distinct from $\mu$ such that $\|\nu\|_{\infty} \leq k$ and $\int_{R} \mu \varphi=\int_{R} \nu \varphi$ for all $\varphi$ in $A(R)$. Using the Infinitesimal Main Inequality, Reich (see [R2]) showed there exists a constant $C=C(k)$ depending only on $k$, such that

$$
\int_{R}|\mu-\nu|^{2}|\varphi| \leq C\left(k\|\varphi\|-\operatorname{Re} \int_{R} \mu \varphi\right),
$$

for all $\varphi$ in $A(R)$. A more general version of this inequality is proved in Section 6. Following Reich's notation $\delta(\varphi)=k\|\varphi\|-\operatorname{Re} \int_{R} \mu \varphi$, we call inequality (6) the Infinitesimal Delta Inequality.

Since $\mu$ and $\nu$ are distinct, there is $\epsilon>0$ and a compact set $E$ in $R$ of positive Lebesgue measure such that $|\mu-\nu| \geq \epsilon$ on $E$. Then, by (6),

$$
\epsilon^{2} \int_{E}|\varphi| \leq C\left(k-\operatorname{Re} \int_{R} \mu \varphi\right),
$$

whenever $\|\varphi\|=1$.

Inequality (7) holds for every unit vector $\varphi$ in $A(R)$. In order to arrive at a contradiction, we need to find a quadratic differential $\varphi$ of norm 1 for which this inequality fails. Strebel's frame mapping theorem provides the existence of a unit vector $\varphi$ in $A(R)$ for every Strebel point in $T$. For arbitrary $r>0$ form the truncation $\alpha$ of $\mu$ to $E$ by setting

$$
\alpha= \begin{cases}\mu & \text { on } E \\ \frac{\mu}{1+r} & \text { on } R \backslash E .\end{cases}
$$

We claim that the hypothesis that $\mu$ is uniquely extremal implies $H(\alpha)<K_{0}(\alpha)$ for arbitrary $r>0$ and arbitrary compact subsets $E$ in $R$ of positive measure. If $s=k /(1+r)$, then clearly the boundary dilatation of $\alpha$ is less than or equal to $(1+s) /(1-s)$. Assume $H(\alpha)=K_{0}(\alpha)$. Then there exists $\eta$ in the class of $\alpha$ with $\|\eta\|_{\infty} \leq s$. Let $f^{\mu}, f^{\alpha}$ and $f^{\eta}$ be quasiconformal homeomorphisms of the unit disk $\Delta$ normalized to fix $1,-1$, and $i$, whose Beltrami differentials are the lifts of $\mu, \alpha$ 
and $\eta$ respectively, and let $F$ be the lift of a compact set $E$. Let $h=f^{\mu} \circ\left(f^{\alpha}\right)^{-1}$. Then the Beltrami coefficient of $h$ is equal to zero on $f^{\alpha}(F)$, and the dilatation $K$ of $h$ on $f^{\alpha}(\Delta-F)$ is equal to $K\left(\left.\mu\right|_{\Delta-F}\right) / K\left(\left.\alpha\right|_{\Delta-F}\right)$. Thus,

$$
K(h) \leq \frac{K(\mu)}{K\left(\frac{\mu}{1+r}\right)} .
$$

Since $\alpha$ and $\eta$ are in the same class in $T$, the pull-backs of the Beltrami coefficients of $f^{\mu}=h \circ f^{\alpha}$ and $h \circ f^{\eta}$ are in the same class in T. Furthermore,

$$
K\left(h \circ f^{\eta}\right) \leq K(h) K\left(f^{\eta}\right) \leq \frac{K\left(f^{\mu}\right)}{K\left(f^{\mu /(1+r)}\right)} K\left(f^{\eta}\right) \leq K\left(f^{\mu}\right) .
$$

By the unique extremality of $\mu$ in its class in $T$, the Beltrami coefficient of $h \circ f^{\eta}$ is equal to $\mu$. Thus, from the normalization, $h \circ f^{\eta}=f^{\mu}$. Hence $f^{\eta}=h^{-1} \circ f^{\mu}=f^{\alpha}$, $\eta=\alpha$, but $\|\eta\|_{\infty} \leq s<k=\|\alpha\|_{\infty}$.

Therefore, the extremal dilatation $K_{0}([\alpha])$ is strictly greater than the boundary dilatation $H([\alpha])$. Thus, $[\alpha]$ is a Strebel point in $T$; and by Strebel's frame mapping theorem there exists $s_{r}=k_{0}([\alpha])>s$ and a unit vector $\varphi$ in $A(R)$ such that $\alpha$ and $s_{r}|\varphi| / \varphi$ are equivalent in $T$. Therefore, by the Reich-Strebel second fundamental inequality (3),

$$
\frac{1+s}{1-s}<\frac{1+s_{r}}{1-s_{r}} \leq \int_{R}|\varphi| \frac{\left|1+\alpha \frac{\varphi}{|\varphi|}\right|^{2}}{1-|\alpha|^{2}} .
$$

Hence, if $\mu_{1}=\mu /(1+r)$, then

$$
\begin{aligned}
\frac{1+s}{1-s} & <\int_{R-E}|\varphi| \frac{\left|1+\mu_{1} \frac{\varphi}{|\varphi|}\right|^{2}}{1-\left|\mu_{1}\right|^{2}}+\int_{E}|\varphi| \frac{\left|1+\mu \frac{\varphi}{|\varphi|}\right|^{2}}{1-|\mu|^{2}} \\
& \leq \int_{R}|\varphi| \frac{1+\left.\mu_{1} \frac{\varphi}{|\varphi|}\right|^{2}}{1-\left|\mu_{1}\right|^{2}}+C_{1}(k) r \int_{E}|\varphi| .
\end{aligned}
$$

Thus,

$$
\begin{gathered}
\frac{1+s}{1-s}<\frac{1+s^{2}+2 \operatorname{Re} \int_{R} \mu_{1} \varphi}{1-s^{2}}+C_{1}(k) r \int_{E}|\varphi|, \\
2\left(s-\operatorname{Re} \int_{R} \mu_{1} \varphi\right) \leq C_{1}(k) r \int_{E}|\varphi| \\
k-\operatorname{Re} \int_{R} \mu \varphi \leq C_{2}(k) r \int_{E}|\varphi| .
\end{gathered}
$$


Therefore, (7) and (8) yield

$$
\epsilon^{2} \int_{E}|\varphi| \leq C_{3}(K) r \int_{E}|\varphi|
$$

which is a contradiction provided that $r$ is sufficiently small.

Remark. The truncation of a Beltrami coefficient was used in [L1] to show that Strebel points are dense in Teichmüller space. It was again used in [ELa] and [MM].

We now prove the converse of Lemma 1, using similar methods and interchanging the corresponding global and infinitesimal arguments from the proof of Lemma 1.

Lemma 2. If $\mu$ has constant absolute value and is uniquely extremal in its class in $B$, then $\mu$ is uniquely extremal in its class in $T$.

Proof. Let $\mu$ be uniquely extremal in its infinitesimal Teichmüller class with $|\mu(z)| \equiv k$. Suppose that $\mu$ is not uniquely extremal in its Teichmüller class. Then there exists a Beltrami differential $\nu$ distinct from $\mu$ such that $\nu$ and $\mu$ belong to the same class in $T$ and $\|\nu\|_{\infty} \leq k$. Let $f$ and $g$ be quasiconformal mappings with domain $R$ and Beltrami coefficients $\mu$ and $\nu$ respectively, which agree on the ideal boundary of $R$. Let $\tilde{\mu}$ be the Beltrami coefficient of $f^{-1}$, and let $\tilde{\nu}$ be the Beltrami coefficient of $g^{-1}$. The Main Inequality yields a constant $C=C(k)$ depending only on $k$, such that

$$
\int_{R}\left|\frac{\tilde{\mu}(f)-\tilde{\nu}(f)}{1-\overline{\tilde{\mu}(f)} \tilde{\nu}(f)}\right|^{2}|\varphi| \leq C\left(k \| \varphi||-\operatorname{Re} \int_{R} \mu \varphi\right)
$$

for all $\varphi$ in $A(R)$ (see [R1] and [R2] for more details). In a manner parallel to (6), we refer to the inequality (9) as the Delta Inequality.

Since $\mu$ and $\nu$ are distinct, there is $\epsilon>0$ and a compact set $E$ in $R$ of positive Lebesgue measure such that $|\tilde{\mu}(f)-\tilde{\nu}(f)| \geq \epsilon$ on $E$. Then, by (9),

$$
\frac{\epsilon^{2}}{4} \int_{E}|\varphi| \leq C\left(k-\operatorname{Re} \int_{R} \mu \varphi\right),
$$

whenever $\|\varphi\|=1$. Following the same line of proof as in Lemma 1, we define a new Beltrami coefficient $\alpha$ on $R$ by letting $\alpha(z)$ equal $\mu(z)$ on $E$, and

$$
\alpha(z)=\frac{1}{1+r} \mu(z) \quad \text { for all } z \in R-E .
$$

Suppose that $\alpha$ is equivalent in $B$ to some Beltrami differential $\eta$ with $\|\eta\|_{\infty} \leq$ $k /(1+r)$. Then $\mu$ is equivalent to $\eta+\mu-\alpha$, and

$$
\|\eta+\mu-\alpha\|_{\infty} \leq\|\eta\|_{\infty}+\|\mu-\alpha\|_{\infty} \leq k,
$$


contradicting the unique extremality of $\mu$ in its infinitesimal Teichmüller class. Therefore, the infinitesimal Teichmüller norm of the class of $\alpha$ in $B$ is strictly greater than its boundary semi-norm. Thus, there exists a unit vector $\varphi$ in $A(R)$ such that $\int_{R} \varphi \alpha>k /(1+r)$. Therefore

$$
\frac{k}{1+r}<\int_{E} \varphi \mu+\int_{R-E} \varphi \frac{\mu}{1+r}
$$

Thus,

$$
k-\operatorname{Re} \int_{R} \mu \varphi \leq k r \int_{E}|\varphi|,
$$

and that contradicts inequality (10), provided that $r$ is sufficiently small. (Note that, following the proof of Lemma 1 , one may use the infinitesimal frame mapping theorem to show that there exists a real number $s_{r}$ strictly greater than $k /(1+r)$ and a unit vector $\varphi$ in $A(R)$ such that $\alpha$ and $s_{r}|\varphi| / \varphi$ belong to the same class in $B$ and again conclude that $\int_{R} \varphi \alpha>k /(1+r)$.)

Hamilton, Krushkal, Reich and Strebel (see [H], [K], [RS], and Chapter 6 in [G]) gave a necessary and sufficient condition for a Beltrami coefficient $\mu$ to be extremal in its class: $\mu$ is extremal if, and only if, there exists a sequence of unit vectors $\varphi_{n}$ in $A(R)$ such that

$$
\|\mu\|_{\infty}-\operatorname{Re} \int_{R} \mu \varphi_{n} \rightarrow 0 .
$$

The condition for unique extremality of a Beltrami coefficient $\mu$ with constant modulus given in the following theorem is a further restriction.

Theorem 1. Let $\mu$ be a Beltrami coefficient in $M(R)$ with constant absolute value. Then the following six conditions are equivalent:

(a) $\mu$ is uniquely extremal in its class in $T$;

(b) $\mu$ is uniquely extremal in its class in $B$;

(c) for every measurable subset $E$ of $R$ with nonzero measure, there exists a sequence of unit vectors $\varphi_{n}$ in $A(R)$ such that

$$
\frac{1}{\int_{E}\left|\varphi_{n}\right|}\left(\|\mu\|_{\infty}-\operatorname{Re} \int_{R} \mu \varphi_{n}\right) \rightarrow 0
$$

(d) for every measurable subset $E$ of $R$ with nonzero measure, there exists a sequence of unit vectors $\varphi_{n}$ in $A(R)$ such that

$$
\frac{1}{\int_{E}\left|\varphi_{n}\right|} \operatorname{Re} \int_{E} \mu \varphi_{n} \rightarrow\|\mu\|_{\infty}
$$


and

$$
\frac{1}{\int_{E}\left|\varphi_{n}\right|}\left(\|\mu\|_{\infty} \int_{R-E}\left|\varphi_{n}\right|-\operatorname{Re} \int_{R-E} \mu \varphi_{n}\right) \rightarrow 0
$$

(e) $\mu$ is extremal in its class in $T(R)$ and, for every compact subset $E$ of $R$ with nonzero measure and every $r>0,\left[\mu_{E}+\frac{1}{1+r} \mu \chi_{R-E}\right]$ is a Strebel point in $T ; ;^{*}$

(f) $\mu$ is extremal in its class in $B(R)$ and, for every compact subset $E$ of $R$ with nonzero measure and every $r>0,\left[\mu_{E}+\frac{1}{1+r} \mu \chi_{R-E}\right]$ is an infinitesimal Strebel point, that is,

$$
b\left(\left[\mu \chi_{E}+\frac{1}{1+r} \mu \chi_{R-E}\right]\right)<\left\|\left[\mu \chi_{E}+\frac{1}{1+r} \mu \chi_{R-E}\right]\right\|
$$

(g) $\mu$ satisfies Reich's condition on $R$.

Proof. (a) implies (b) by Lemma 1, and (b) implies (a) by Lemma 2. Suppose that (a) holds and let $E$ be any measurable subset of $R$ with positive measure. Take a compact subset $F$ of $E$ with positive measure. Inequality (8) in the proof of Lemma 1 yields the existence of a sequence of unit vectors $\varphi_{n}$ in $A(R)$ such that

$$
\|\mu\|_{\infty}-\operatorname{Re} \int_{R} \mu \varphi_{n} \leq \frac{C_{2}(K)}{n} \int_{F}\left|\varphi_{n}\right| \leq \frac{C_{2}(K)}{n} \int_{E}\left|\varphi_{n}\right| .
$$

Furthermore,

$$
\frac{1}{\int_{E}\left|\varphi_{n}\right|}\left(\|\mu\|_{\infty}-\operatorname{Re} \int_{R} \mu \varphi_{n}\right) \geq \frac{1}{\int_{E}\left|\varphi_{n}\right|}\left(\|\mu\|_{\infty}-\|\mu\|_{\infty} \int_{R}\left|\varphi_{n}\right|\right) \geq 0
$$

for all $n$. Thus (a) implies (c). Inequality (7) shows that (c) implies (b). Since

$$
\begin{array}{r}
\frac{1}{\int_{E}\left|\varphi_{n}\right|}\left(\|\mu\|_{\infty}-\operatorname{Re} \int_{R} \mu \varphi_{n}\right)= \\
\frac{1}{\int_{E}\left|\varphi_{n}\right|}\left(\|\mu\|_{\infty} \int_{R-E}\left|\varphi_{n}\right|-\operatorname{Re} \int_{R-E} \mu \varphi_{n}\right) \\
+\frac{1}{\int_{E}\left|\varphi_{n}\right|}\left(\|\mu\|_{\infty} \int_{E}\left|\varphi_{n}\right|-\operatorname{Re} \int_{E} \mu \varphi_{n}\right),
\end{array}
$$

(c) is equivalent to (d). The third paragraph of the proof of Lemma 1 shows that (a) implies (e). Suppose that (e) holds. If $[\mu]$ is a Strebel point, then (c) holds for a constant sequence $\varphi_{n}$. If $[\mu]$ is a non-Strebel point, the fourth paragraph of the proof of Lemma 1 shows we have inequality (8); thus (e) implies (c). Similarly, the proof of Lemma 2 yields $(b) \Rightarrow(f) \Rightarrow(c)$. We prove the equivalence of $(b)$ and (g) in Section 11 (see Theorem 13).

\footnotetext{
${ }^{*} \chi$ is the characteristic function of a set.
} 
Remark. In [R3] Reich showed that there exists a Beltrami coefficient $\mu$ with constant absolute value such that $\mu$ satisfies (12), the first half of condition (d), but is not uniquely extremal in its class in $B$. Therefore, the second half (13) of the condition (d) cannot be removed from Theorem 1.

\section{Uniquely extremal differentials of Teichmüller type}

If $K=(1+k) /(1-k)>1$ and $f(z)$ is the affine stretch $f(z)=K x+i y$ defined on a plane domain $\Omega$, then the Beltrami coefficient $\mu$ of $f$ has the form

$$
\mu(z)=k \frac{\left|\varphi_{0}\right|}{\varphi_{0}} \quad \text { with } \varphi_{0} \equiv 1 .
$$

In [R4], Reich showed that if there exists a sequence $\varphi_{n}$ in $A(\Omega)$ such that

(1) $\varphi_{n}(z) \rightarrow \varphi_{0}(z)$ for all $z$ in $\Omega$, and

(2) $\left\|\varphi_{n}\right\|-\operatorname{Re} \int_{\Omega} \varphi_{n} \rightarrow 0$,

then $f(z)$ is uniquely extremal. (A similar proof can be obtained by using Theorem 1.) In [R4], Reich showed that a sequence $\varphi_{n}(z)=e^{-z / n}$ in $A(\Omega)$ satisfies conditions (1) and (2) for $\Omega=\Omega_{\alpha}=\left\{z: x>|y|^{\alpha}\right\}$ with $\alpha>3$, and asked if the conditions (1) and (2) are not only sufficient but also necessary for the unique extremality of $f$. The next theorem provides an affirmative answer to that question.

Theorem 2. Let $\varphi$ be a nonzero holomorphic quadratic differential on a Riemann surface $R$, and let $f$ be a quasiconformal mapping with domain $R$ and Beltrami coefficient $\mu=k|\varphi| / \varphi, 0<k<1$. Then $f$ is uniquely extremal if and only if there exists a sequence $\varphi_{n}$ in $A(R)$ such that

(1) $\varphi_{n}(z)$ converges to $\varphi(z)$ uniformly on compact subsets of $R$, and

(2) $k\left\|\varphi_{n}\right\|-\operatorname{Re} \int_{R} \varphi_{n} \mu \rightarrow 0$.

Proof. If there exists a sequence $\varphi_{n}$ satisfying conditions (1) and (2), then Fatou's lemma implies that the sequence $\varphi_{n} /\left\|\varphi_{n}\right\|$ satisfies the condition (c) of Theorem 1 for every measurable subset $E$ of $R$. Thus, $f$ is uniquely extremal in its Teichmüller class.

To prove the converse, suppose that $f$ is uniquely extremal and pick a conformal disc $E \subset R$, that is, an open set $E$ whose closure $\bar{E}$ is in a single coordinate patch (with local coordinate $z$ ) such that $\bar{E}$ is a closed disc in the $z$-plane. Since the zeros of $\varphi$ form a discrete set, we can change the radius of $z(E)$ by a small amount so that $\varphi$ has no zeros on the boundary of $E$. By condition (c) of Theorem 1, there exists a sequence $\psi_{n}$ of unit vectors in $A(R)$ such that

$$
\frac{k-\operatorname{Re} \int_{R} \psi_{n} \mu}{\int_{E}\left|\psi_{n}\right|} \rightarrow 0
$$


If

$$
\varphi_{n}=\frac{\psi_{n}}{\int_{E}\left|\psi_{n}\right|} \int_{E}|\varphi|
$$

then $\int_{E}\left|\varphi_{n}\right|=\int_{E}|\varphi|$ and

$$
0 \leq k\left\|\varphi_{n}\right\|-\operatorname{Re} \int_{R} \varphi_{n} \mu=\int_{E}|\varphi| \frac{k-\operatorname{Re} \int_{R} \psi_{n} \mu}{\int_{E}\left|\psi_{n}\right|} \rightarrow 0 .
$$

Hence,

$$
0 \leq k \int_{E}\left|\varphi_{n}\right|-\operatorname{Re} \int_{E} \varphi_{n} \mu \leq k|| \varphi_{n}||-\operatorname{Re} \int_{R} \varphi_{n} \mu \rightarrow 0
$$

Since $\varphi$ is holomorphic in a neighborhood of $\bar{E}$ and has no zeros on the boundary of $E, A(E)$ is weakly uniformly convex at $\varphi / \int_{E}|\varphi|$ (see [Y] and [L2]). Thus $\int_{E}\left|\varphi_{n}-\varphi\right| \rightarrow 0$. Hence $\varphi_{n}$ converges to $\varphi$ uniformly on compact subsets of $E$.

Let $V_{1}=E$ and $F$ be a conformal disc in $R$ such that $F \cap E$ is not empty. We have

$$
\begin{aligned}
0 & \leq k-\operatorname{Re} \int_{F} \frac{\varphi_{n}}{\int_{F}\left|\varphi_{n}\right|} \mu=\frac{k \int_{F}\left|\varphi_{n}\right|-\operatorname{Re} \int_{F} \varphi_{n} \mu}{\int_{F}\left|\varphi_{n}\right|} \\
& \leq \frac{k \int_{R}\left|\varphi_{n}\right|-\operatorname{Re} \int_{R} \varphi_{n} \mu}{\int_{F \cap E}\left|\varphi_{n}\right|} \rightarrow 0 \quad \text { because } \int_{F \cap E}\left|\varphi_{n}\right| \rightarrow \int_{F \cap E}|\varphi|>0 .
\end{aligned}
$$

Therefore, if the boundary of $F$ contains no zeros of $\varphi$,

$$
\int_{F}\left|\frac{\varphi_{n}}{\int_{F}\left|\varphi_{n}\right|}-\frac{\varphi}{\int_{F}|\varphi|}\right| \rightarrow 0
$$

Let $p \in F \cap E$. Then

$$
\frac{\varphi_{n}(p)}{\int_{F}\left|\varphi_{n}\right|}-\frac{\varphi(p)}{\int_{F}|\varphi|} \rightarrow 0
$$

and $\varphi_{n}(p) \rightarrow \varphi(p)$ in any local coordinate of $p$. Thus $\int_{F}\left|\varphi_{n}\right| \rightarrow \int_{F}|\varphi|$. Hence

$$
\int_{F}\left|\varphi_{n}-\varphi\right| \rightarrow 0
$$

Therefore, $\varphi_{n}$ converges to $\varphi$ uniformly on compact subsets of $F$. Thus, $\varphi_{n}$ converges to $\varphi$ uniformly on compact subsets of $V=V_{1} \cup V_{2} \cup V_{3} \cup \cdots$, where $V_{n+1}$ is the union of all conformal discs in $R$ that intersect $V_{n}$. Clearly $V$ is open, and since every point in $R$ is the center of some conformal disc in $R, V$ is closed. Therefore $V$ is both open and closed; and since $R$ is a connected set, $V \equiv R$. 


\section{Delta Inequalities}

In this section we generalize Reich's Delta Inequality (9) and Reich's Infinitesimal Delta Inequality (6) to an arbitrary extremal Beltrami coefficient $\mu$. We start with the generalization of the Delta Inequality.

Let $\mu$ and $\nu$ be two equivalent Beltrami coefficients in $T$, with

$$
\|\nu\|_{\infty} \leq k=\|\mu\|_{\infty}<1
$$

and let $\varphi$ be a nonzero vector in $A(R)$. Then there exist homotopic quasiconformal mappings $f$ and $g$ defined on $R$ with Beltrami coefficients $\mu$ and $\nu$, respectively, such that $f(R)=g(R)$ and $f(p)=g(p)$ for every point $p$ on the ideal boundary of $R$. Let $\tilde{\mu}$ and $\tilde{\nu}$ be the Beltrami coefficients of the quasiconformal mappings $f^{-1}$ and $g^{-1}$, respectively.

Theorem 3. If $\mu$ and $\nu$ are equivalent Beltrami coefficients in $T$ with

$$
\|\nu\|_{\infty} \leq k=\|\mu\|_{\infty}<1
$$

then

$$
\int_{R}\left|\frac{\tilde{\mu}(f)-\tilde{\nu}(f)}{1-\overline{\tilde{\mu}(f)} \tilde{\nu}(f)}\right|^{2}|\varphi| \leq C\left(k\|\varphi\|-\operatorname{Re} \int_{R} \mu \varphi\right),
$$

for all quadratic differentials $\varphi$ in $A(R)$. The constant $C$ depends only on $k=\|\mu\|_{\infty}$.

Proof. Let $\alpha(z)$ and $\beta(z)$ be the Beltrami coefficients of the quasiconformal mappings $f^{-1}$ and $g^{-1}$, respectively, evaluated at the point $f(z)$, that is, $\alpha(z)=$ $\tilde{\mu}(f(z))$ and $\beta(z)=\tilde{\nu}(f(z))$. Let $\rho=|\alpha-\beta|^{2}$,

$$
I=I(\varphi)=\int_{R} \frac{\rho}{|1-\bar{\alpha} \beta|^{2}}|\varphi|,
$$

and $\delta=\delta(\varphi)=k\|\varphi\|-\operatorname{Re} \int_{R} \mu \varphi$. Also let

$$
Q=\left(1-|\alpha|^{2}\right)\left(1-|\beta|^{2}\right) \quad \text { and } P=(\alpha-\beta)(1-\alpha \bar{\beta}) \alpha^{-1} .
$$

Suppose first that $|\mu(z)|$ is bounded from below by a positive constant $s$ for almost every $z$ in $R$. Then for any $\varphi \in A(R)$ we have as an easy consequence of the Main Inequality

$$
\operatorname{Re} \int_{R} \frac{P}{Q} \mu \varphi \leq \int_{R} \frac{\rho}{Q}|\varphi| .
$$

In order to get an estimate involving $\delta(\varphi)$, add

$$
l=\int_{R}|\alpha| \operatorname{Re} \frac{P}{Q}|\varphi|
$$


to both sides. We get

$$
\int_{R} \frac{|\alpha| \operatorname{Re} P-\rho}{Q}|\varphi| \leq \operatorname{Re} \int_{R} \frac{P}{Q}(|\alpha||\varphi|-\mu \varphi) .
$$

Furthermore,

$$
\operatorname{Re} P=|\alpha|^{-2} \operatorname{Re}\left((\alpha-\beta)\left(\bar{\alpha}-|\alpha|^{2} \bar{\beta}\right)\right)=|\alpha|^{-2}\left(\rho+\left(1-|\alpha|^{2}\right)\left(\operatorname{Re} \alpha \bar{\beta}-|\beta|^{2}\right)\right) .
$$

Hence

$$
\operatorname{Re} P-\rho=|\alpha|^{-2}\left(1-|\alpha|^{2}\right)\left(|\alpha|^{2}-\operatorname{Re} \alpha \bar{\beta}\right) .
$$

Thus

$$
|\alpha| \operatorname{Re} P-\rho=A \rho+B(|\alpha|-|\beta|)
$$

where $A=2^{-1}|\alpha|^{-1}(1-|\alpha|)^{2}, B=2^{-1}|\alpha|^{-1}(|\alpha|+|\beta|)\left(1-|\alpha|^{2}\right)$.

Since $|\mu|$ is bounded from below by a positive constant $s>0, A$ and $B$ are bounded away from zero and infinity. Thus, using (15) and the estimate $|\beta|-|\alpha| \leq$ $k-|\mu|$, (14) yields a constant $c$ which depends only on $k=\|\mu\|_{\infty}$ and $s$ such that

$$
I \leq c\left(I_{1}+\tau\{\varphi\}\right)
$$

where

$$
I_{1}=\int_{R} \sqrt{\rho}|| \mu \varphi|-\mu \varphi|
$$

and

$$
\tau=\tau\{\varphi\}=\int_{R}(k-|\mu|)|\varphi| .
$$

Using the Cauchy-Schwarz inequality and the identity

$$
|| w|-w|^{2}=2|w|(|w|-\operatorname{Re} w)
$$

we obtain

$$
I_{1} \leq c_{1} I^{1 / 2} \delta^{1 / 2} .
$$

Using this and the inequality $\tau \leq \delta$, we get

$$
I \leq c_{2}\left(I^{1 / 2} \delta^{1 / 2}+\delta\right) .
$$

On letting $x$ be the positive number $I^{1 / 2} / \delta^{1 / 2}$, this inequality becomes $x \leq c_{2}(1+1 / x)$, which implies $x$ is bounded. We conclude that

$$
I \leq C(k, s) \delta
$$


Now assume that $\mu$ and $\nu$ are two arbitrary equivalent Beltrami coefficients such that $\|\nu\|_{\infty} \leq\|\mu\|_{\infty}=k<1$. Note that $I(\varphi)$ is invariant under postcompositions by quasiconformal mappings. Fix a unit vector $q$ in $A(R)$ and let

$$
E=\{z \in f(R):|\tilde{\mu}(z)|<k / 2\} .
$$

Take $z \in E$. If $\tilde{\mu}(z) \neq \tilde{\nu}(z)$, let $\eta(z)$ be the point on the hyperbolic circle

$$
S=\left\{w \in \Delta: \rho(w, \tilde{\mu}(z))=\frac{1}{2} \rho(0, k / 2)\right\}
$$

with minimal distance from $\tilde{\nu}(z)$ ( $\rho$ is the hyperbolic distance in the unit disc $\Delta$ ). If $\tilde{\mu}(z)=\tilde{\nu}(z) \neq 0$, let $\eta(z)=a \tilde{\mu}(z)$, where $a$ is a positive constant so that $\eta(z) \in S$. Finally, if $\tilde{\mu}(z)=\tilde{\nu}(z)=0$, let

$$
\eta(z)=\frac{k|q(z)|}{2 q(z)}
$$

Note that this definition is independent of the local coordinate chart containing the point $z$. Extend $\eta$ by setting $\eta(z)=0$ for all $z$ in the complement of $E$ in $f(R)$. Let $h$ be a quasiconformal mapping with domain $f(R)$ and Beltrami coefficient $\eta$. Then the Beltrami coefficients $\mu_{1}$ and $\nu_{1}$ of $h \circ f$ and $h \circ g$ are equivalent. Furthermore,

$$
\left|\mu_{1}(z)\right|=\left|\frac{\eta(f z)-\tilde{\mu}(f z)}{1-\overline{\eta(f z)} \tilde{\mu}(f z)}\right|
$$

and

$$
\left|\nu_{1}(z)\right|=\left|\frac{\eta(g z)-\tilde{\nu}(g z)}{1-\overline{\eta(g z)} \tilde{\nu}(g z)}\right| .
$$

Hence, $\rho\left(0, \mu_{1}\right)=\rho(\eta(f), \tilde{\mu}(f))$ and $\rho\left(0, \nu_{1}\right)=\rho(\eta(g), \tilde{\nu}(g))$. The definition of $\eta(z)$ yields $\left|\mu_{1}(z)\right| \geq k / 2$ and $\left|\nu_{1}(z)\right| \leq k$ for almost all $z \in R$. Also $\left|\mu_{1}(z)\right|=k / 2$ for almost all $z \in f^{-1}(E)$ and $\left|\mu_{1}(z)\right|=|\mu(z)| \geq k / 2$ for all $z \in R-f^{-1}(E)$. Since

$$
(h \circ g)^{-1} \circ(h \circ f)=g^{-1} \circ f \text {, }
$$

we have

$$
I(\varphi) \leq C(k, k / 2)\left(k\|\varphi\|-\operatorname{Re} \int_{R} \mu_{1} \varphi\right) .
$$

Let $G=f^{-1}(E)$. Then

$$
3 \operatorname{Re} \int_{G} \mu \varphi-\operatorname{Re} \int_{G} \mu_{1} \varphi \leq(3 k / 2+k / 2) \int_{G}|\varphi|=2 k \int_{G}|\varphi| .
$$

Hence

$$
3 \operatorname{Re} \int_{R} \mu \varphi \leq 2 k \int_{G^{c}}|\varphi|+\operatorname{Re} \int_{G^{c}} \mu \varphi+2 k \int_{G}|\varphi|+\operatorname{Re} \int_{G} \mu_{1} \varphi .
$$


That yields

$$
k\|\varphi\|-\operatorname{Re} \int_{R} \mu_{1} \varphi \leq 3\left(k\|\varphi\|-\operatorname{Re} \int_{R} \mu \varphi\right)=3 \delta(\varphi) .
$$

Therefore, $I(\varphi) \leq 3 C(k, k / 2) \delta(\varphi)$; and that proves the theorem.

We now generalize the Infinitesimal Delta Inequality.

Theorem 4. There exists a universal constant $C$ such that for every pair of infinitesimally equivalent Beltrami coefficients $\mu$ and $\nu$ with

$$
\|\nu\|_{\infty} \leq\|\mu\|_{\infty}<\infty
$$

we have

$$
\int_{R}|\mu-\nu|^{2}|\varphi| \leq C\|\mu\|_{\infty}\left(\|\mu\|_{\infty}\|\varphi\|-\operatorname{Re} \int_{R} \mu \varphi\right)
$$

for all quadratic differentials $\varphi$ in $A(R)$. The constant $C$ is independent of $\mu$ and $\nu$.

Proof. First, let $\mu$ and $\nu$ be two equivalent Beltrami coefficients in $B$ with

$$
\|\nu\|_{\infty} \leq k=\|\mu\|_{\infty}<1
$$

and let $\varphi$ be a nonzero vector in $A(R)$. By inequality (4) in Section 3,

$$
\operatorname{Re} \int_{R} \frac{(1-\bar{\nu} \mu)(\mu-\nu)}{1-|\nu|^{2}} \varphi \leq \int_{R} \frac{|\mu-\nu|^{2}}{1-|\nu|^{2}}|\varphi|
$$

Adding

$$
\int_{R} \frac{\operatorname{Re} \frac{(1-\bar{\nu} \mu)(\mu-\nu)}{\mu}}{1-|\nu|^{2}}|\mu||\varphi|
$$

to both sides of this inequality, we obtain

$$
\begin{aligned}
& \int_{R}\left(|\mu| \operatorname{Re} \frac{(1-\bar{\nu} \mu)(\mu-\nu)}{\mu}-|\mu-\nu|^{2}\right) \frac{1}{1-|\nu|^{2}}|\varphi| \\
& \leq \operatorname{Re} \int_{R} \frac{(1-\bar{\nu} \mu)(\mu-\nu)}{\mu} \frac{1}{1-|\nu|^{2}}(|\mu||\varphi|-\mu \varphi) .
\end{aligned}
$$

Suppose first that $\mu$ is bounded below by $s>0$, that is, $|\mu(z)| \geq s>0$ for almost all $z$ in $R$. Since

$$
|\mu| \operatorname{Re} \frac{(1-\bar{\nu} \mu)(\mu-\nu)}{\mu}-|\mu-\nu|^{2}=A|\mu-\nu|^{2}+B(|\mu|-|\nu|),
$$

where

$$
A=\frac{(1-|\mu|)^{2}}{2|\mu|} \geq \frac{1-k^{2}}{2 k}
$$


and

$$
B=\frac{(|\mu|+|\nu|)\left(1-|\mu|^{2}\right)}{2|\mu|} \leq \frac{k}{s},
$$

this together with the estimate $|\nu|-|\mu| \leq k-|\mu|$ and the inequality (6) yields

$$
\int_{R}|\mu-\nu|^{2}|\varphi| \leq c(k, s)\left(\int_{R} \sqrt{|\mu-\nu|^{2}}|| \mu \varphi|-\mu \varphi|+\int_{R}(k-|\mu|)|\varphi|\right) .
$$

Therefore, again using the Cauchy-Schwarz inequality and the identity

$$
|| w|-w|^{2}=2|w|(|w|-\operatorname{Re} w)
$$

we obtain, as in the proof of the Delta Inequality,

$$
\int_{R}|\mu-\nu|^{2}|\varphi| \leq C(k, s)\left(k|| \varphi||-\operatorname{Re} \int_{R} \mu \varphi\right),
$$

where $C(k, s)$ is a constant that depends only on $k$ and $s$.

Now suppose that $\mu$ and $\nu$ are two arbitrary infinitesimally equivalent Beltrami coefficients on a Riemann surface $R$ such that $\|\nu\|_{\infty} \leq\|\mu\|_{\infty}=k<1$, and let $\varphi$ be an arbitrary nonzero vector in $A(R)$. Let $E=\{z \in R:|\mu(z)|<k / 2\}$. Then $(\nu-\mu) / 3$ is an infinitesimally trivial Beltrami coefficient. Thus the Beltrami coefficients

$$
\mu_{1}=\mu \chi_{E^{c}}+\frac{k|\varphi|}{2 \varphi} \chi_{E}
$$

and

$$
\nu_{1}=\mu_{1}+\frac{\nu-\mu}{3}
$$

are infinitesimally equivalent. Clearly, $\left\|\mu_{1}\right\|_{\infty}=k,\left|\mu_{1}(z)\right| \geq k / 2$ for almost all $z$ in $R$, and

$$
\begin{aligned}
\left\|\nu_{1}\right\|_{\infty} & \leq \max \left\{\left\|\frac{2}{3} \mu+\frac{1}{3} \nu\right\|_{E^{c}}, k / 2+\left\|\frac{1}{3}(\nu-\mu)\right\|_{E}\right\} \\
& \leq \max \left\{k, k / 2+\frac{k+k / 2}{3}\right\}=k .
\end{aligned}
$$

Thus

$$
\int_{R}\left|\mu_{1}-\nu_{1}\right|^{2}|\varphi| \leq \frac{C(k)}{9}\left(k|| \varphi \|-\operatorname{Re} \int_{R} \mu_{1} \varphi\right),
$$

where $C(k)=9 C(k, k / 2)$. Furthermore,

$$
k\|\varphi\|-\operatorname{Re} \int_{R} \mu_{1} \varphi=k\|\varphi\|-\operatorname{Re} \int_{E^{\mathrm{c}}} \mu \varphi-\frac{1}{2} k \operatorname{Re} \int_{E}|\varphi| \leq k\|\varphi\|-\operatorname{Re} \int_{R} \mu \varphi,
$$

and

$$
\int_{R}\left|\mu_{1}-\nu_{1}\right|^{2}|\varphi|=\frac{1}{9} \int_{R}|\mu-\nu|^{2}|\varphi|
$$


Therefore,

$$
\int_{R}|\mu-\nu|^{2}|\varphi| \leq C(k)\left(k\|\varphi\|-\operatorname{Re} \int_{R} \mu \varphi\right) .
$$

Finally, let $C=2 C\left(\frac{1}{2}\right)$ and suppose that $\mu$ and $\nu$ are two infinitesimally equivalent Beltrami coefficients on $R$ such that

$$
\|\nu\|_{\infty} \leq\|\mu\|_{\infty}<\infty
$$

If $\|\mu\|_{\infty}=0$, then the generalized Infinitesimal Delta Inequality clearly holds. If $\|\mu\|_{\infty}>0$, then

$$
\mu_{2}=\frac{\mu}{2\|\mu\|_{\infty}}
$$

and

$$
\nu_{2}=\frac{\nu}{2\|\mu\|_{\infty}}
$$

are infinitesimally equivalent and satisfy $\left\|\mu_{2}\right\|_{\infty}=\left\|\nu_{2}\right\|_{\infty}=1 / 2$. Thus

$$
\int_{R}\left|\mu_{2}-\nu_{2}\right|^{2}|\varphi| \leq C\left(\frac{1}{2}\right)\left(\frac{1}{2}|| \varphi \|-\operatorname{Re} \int_{R} \mu_{2} \varphi\right) .
$$

Hence

$$
\int_{R}|\mu-\nu|^{2}|\varphi| \leq 2 C(1 / 2)\|\mu\|_{\infty}\left(\|\mu\|_{\infty}\|\varphi\|-\operatorname{Re} \int_{R} \mu \varphi\right),
$$

and that proves the theorem.

Remark. Miroslav Pavlovic has recently found a short and elegant proof of Theorem 4. He has also made further progress in the study of unique extremality ([P]).

\section{The proof of the Equivalence Theorem}

In this section we prove the Equivalence Theorem. First we generalize Lemma 1.

Lemma 3. If $\mu$ is uniquely extremal in its class in $T$, then $\mu$ is uniquely extremal in its class in $B$.

Proof. Let $\mu$ be uniquely extremal in its Teichmüller class with $\|\mu\|_{\infty}=k$. Suppose that $\mu$ is not uniquely extremal in its infinitesimal Teichmüller class. Then there exists a Beltrami differential $\nu$ distinct from $\mu$ such that $\|\nu\|_{\infty} \leq k$ and $\int_{R} \mu \varphi=\int_{R} \nu \varphi$ for all $\varphi$ in $A(R)$. Hence, there exists $\epsilon>0$ and a compact subset $E$ of $R$, such that $|\mu(z)-\nu(z)| \geq \epsilon$ for almost all $z \in E$.

We divide the proof of Lemma 3 into three steps. 
Step I. The set $F=\{z \in E:|\mu(z)|=k\}$ has measure 0 .

Proof. If $F$ has positive measure, then by the proof of Lemma 1, for every positive real number $r$ there exists a unit vector $\varphi=\varphi(r)$ such that

$$
\delta(\varphi)=k-\operatorname{Re} \int_{R} \mu \varphi \leq C_{2}(K) r \int_{F}|\varphi| .
$$

On the other hand, the generalized Infinitesimal Delta Inequality yields $\epsilon^{2} \int_{F}|\varphi| \leq C k \delta(\varphi)$, which contradicts (17) for $r$ sufficiently small.

Therefore, we may assume that for some $s<k$, the set $G=\{z \in E:|\mu(z)| \leq s\}$ has positive measure.

Step II. If $\eta$ is any Beltrami differential supported in $G$ with $\|\eta\|_{\infty} \leq k$, then there is a positive number $r$ such that the Beltrami coefficient $\xi(r)=$ $r\left(\mu \chi_{R-G}+\eta \chi_{G}\right)$ is uniquely extremal in its class in $T$.

Proof. Choose a positive number $r$ such that $\rho(0, r k)+\rho(0, s) \leq \rho(r k, k)$, where $\rho$ is the Poincaré metric in the unit disk. Let $\beta$ be a Beltrami differential on $R$ such that $\beta$ and $\xi(r)$ belong to the same class in $T$ and $\|\beta\|_{\infty} \leq r k$. Let $f, g$ and $h$ be normalized quasiconformal homeomorphisms with domain $R$ and Beltrami coefficients $\xi(r), \beta$, and $\mu$. Then $f$ and $g$ are homotopic to one another and coincide on the ideal boundary of $R$. Let $j=h \circ f^{-1}$. Then $h=j \circ f$ and $j \circ g$ belong to the same Teichmüller class, and $\frac{1}{2} \ln K(j)$ is less than or equal to $\rho(r k, k)$. Therefore, $K(j \circ g) \leq K(\mu)$; and by the unique extremality of $\mu$ in its class in $T, \xi(r)=\beta$.

Step III. $\mu(z) \equiv \nu(z)$.

Proof. Define a Beltrami coefficient $\eta(z)$ on $R$ in the following way. If $z$ is outside $G$, let $\eta(z)=0$. If $z \in G$, then let $\eta(z)$ be the point on the circle $\{w:|w-\mu(z)|=k\}$ whose distance to $\nu(z)$ is minimal. By Step II, there exists $r>0$ such that $r(\mu-\eta)$ is uniquely extremal in its Teichmüller class. Furthermore, $r(\mu-\eta)$ is equivalent to $r(\nu-\eta)$ and

$$
\|r(\nu-\eta)\|_{\infty}=r\|(\nu-\eta)\|_{\infty} \leq r k .
$$

Also $|r(\mu(z)-\eta(z))|=r k$ for all $z$ in $G$; thus, by Step I,

$$
r(\mu(z)-\eta(z))=r(\nu(z)-\eta(z)) \quad \text { for almost all } z \in G .
$$

Hence $\mu(z)=\nu(z)$ for almost all $z \in G$.

Next we generalize Lemma 2.

Lemma 4. If $\mu$ is uniquely extremal in its class in $B$, then $\mu$ is uniquely extremal in its class in $T$. 
Proof. Let $\mu$ be uniquely extremal in its infinitesimal Teichmüller class with $\|\mu\|_{\infty}=k$. Suppose that $\mu$ is not uniquely extremal in its Teichmüller class. Then there exists a Beltrami differential $\nu$ distinct from $\mu$ but belonging to the same class in $T$ such that $\|\nu\|_{\infty} \leq k$. Let $f$ be a quasiconformal mapping with domain $R$ and Beltrami coefficient $\mu$. Then there exists a quasiconformal mapping $g$ with domain $R$ and Beltrami coefficient $\nu$, such that $f$ and $g$ are homotopic and coincide on the ideal boundary of $R$. Let $\tilde{\mu}$ be the Beltrami coefficient of $f^{-1}$, and let $\tilde{\nu}$ be the Beltrami coefficient of $g^{-1}$. Clearly $|\mu| \equiv|\tilde{\mu}(f)|$ and $|\nu| \equiv|\tilde{\nu}(g)|$. It is enough to prove that $\tilde{\mu} \equiv \tilde{\nu}$. If $\tilde{\mu}$ and $\tilde{\nu}$ do not coincide, there exist $\epsilon>0$ and a compact subset $E$ of $R$ such that

$$
\frac{|\tilde{\mu}(f(z))-\tilde{\nu}(f(z))|}{\mid 1-\tilde{\mu}(f(z)) \overline{\tilde{\nu}(f(z)) \mid}} \geq \epsilon
$$

for almost all $z \in E$. The proof of Lemma 4 now follows the same steps as those in the proof of Lemma 3.

Step I. The set $F=\{z \in E:|\mu(z)|=k\}$ has measure 0 .

Proof. If the set $F$ had positive measure, then by the proof of Lemma 2, for every positive real number $r$, there would exist a unit vector $\varphi=\varphi(r)$ such that

$$
\delta(\varphi)=k-\operatorname{Re} \int_{R} \mu \varphi \leq k r \int_{F}|\varphi| .
$$

On the other hand, the generalized Delta Inequality yields $\epsilon^{2} \int_{F}|\varphi| \leq C \delta(\varphi)$, a contradiction provided that $r$ is sufficiently small.

Therefore, we may assume that for some $s<k$, the set $G=\{z \in E:|\mu(z)| \leq s\}$ has positive measure.

Step II. If $\eta$ is any Beltrami differential on $G$ with $\|\eta\|_{\infty} \leq k$, then the Beltrami coefficient $\xi=\mu \chi_{R-G}+\eta \chi_{G}$ is uniquely extremal in its class in $B$.

Proof. Take any real number $C$ such that $C>2 k /(k-s)$. Suppose that some Beltrami differential $\alpha$ is equivalent to $\xi$ in $B$ and $\|\alpha\|_{\infty} \leq k$. Then $C \mu$ and $\alpha+C \mu-\xi$ belong to the same class in $B$. Furthermore,

$$
\|\alpha+C \mu-\xi\|_{\infty} \leq\|\alpha\|_{\infty}+\|C \mu-\xi\|_{\infty} \leq k+\max \{C s+k,(C-1) k\}=C k .
$$

By the unique extremality of $\mu$ in its class in $B$, we have $C \mu=\alpha+C \mu-\xi$. Thus $\alpha=\xi$, and that proves Step II.

Step III. $\tilde{\mu}(f) \equiv \tilde{\nu}(f)$.

Proof. Define a Beltrami coefficient $\eta(z)$ on $f(R)$ in the following way. If $z$ is outside $f(G)$, let $\eta(z)=0$. If $z \in f(G)$, then let $\eta(z)$ be the point on the 
hyperbolic circle $s=\{w \in \Delta: \rho(w, \tilde{\mu}(z))=\rho(0, k)\}$ whose hyperbolic distance to $\tilde{\nu}(z)$ is minimal. Let $h$ be a quasiconformal mapping with domain $f(R)$ and Beltrami coefficient $\eta$. Then the Beltrami coefficients $\mu_{1}$ and $\nu_{1}$ of $h \circ f$ and $h \circ g$ are equivalent in $T$. Furthermore, $\left|\mu_{1}(z)\right|=k$ for all $z$ in $G$, and $\left\|\nu_{1}\right\|_{\infty} \leq\left\|\mu_{1}\right\|_{\infty}=k$. By Step II, $\mu_{1}(z)$ is uniquely extremal in its infinitesimal Teichmüller class; thus $\tilde{\mu_{1}}(z)=\tilde{\nu_{1}}(z)$ for almost all $z \in h(f(G))$ by Step I. Therefore, the quasiconformal mapping

$$
(h \circ g)^{-1} \circ\left((h \circ f)^{-1}\right)^{-1}=g^{-1} \circ f
$$

has dilatation equal to zero on $G$. Hence $\tilde{\nu}(z)=\tilde{\mu}(z)$ for almost all $z \in f(G)$.

Taken together, Lemmas 3 and 4 constitute the Equivalence Theorem:

Theorem 5 (Equivalence Theorem). A Beltrami coefficient $\mu$ is uniquely extremal in its class in $T$ if, and only if, $\mu$ is uniquely extremal in its class in $B$.

\section{The Equivalence Theorem in Teichmüller geometry}

Theorem 6. If a nonzero Beltrami coefficient $\mu$ is uniquely extremal in its class in $T$ and $\lambda$ is a complex number with $|\lambda|<1 /\|\mu\|_{\infty}$, then $\lambda \mu$ is also uniquely extremal in its class in $T$.

Proof. This Theorem follows from the Equivalence Theorem and the fact that the condition for unique extremality in $B$ is invariant under multiplication by $\lambda$.

In [EKK], Earle, Kra and Krushkal showed (see also [Liz]) that the following four conditions are equivalent for every extremal nonzero Beltrami coefficient $\mu$ in $M(R)$.

(a) $\mu$ is uniquely extremal in its Teichmüller class and has constant absolute value (up to a set of measure zero, as usual),

(b) there is only one geodesic segment joining the class of $\mu$ to the basepoint in $T$,

(c) there is only one holomorphic isometry $f$ from the unit disk $\Delta$ into $T$ such that $f(0)=[0]$ and $f\left(\|\mu\|_{\infty}\right)=[\mu]$, and

(d) there is only one holomorphic map $g: \Delta \rightarrow M(R)$ such that $g(0)=0$ and $\mu$ is equivalent to $g\left(\|\mu\|_{\infty}\right)$ in $T$.

Combining this theorem with Theorem 6, we see that conditions (a), (b), (c) and (d) are all equivalent to the same conditions with $\mu$ replaced by $C \mu$ for any complex constant $C$ with $|C|<1 /\|\mu\|_{\infty}$, and, by Theorem 1 , they yield the conditions (b), (c), (d), (e), (f) and (g) of that theorem. In particular, we have the following two theorems. 
Theorem 7. Suppose $\mu$ in $M(R)$ is extremal and nonzero. If there is only one geodesic segment joining $[0]$ and $[\mu]$ in $T(R)$, then there is only one geodesic segment joining $[0]$ and $[\lambda \mu]$, where $\lambda$ is any complex constant with $|\lambda|<1 /\|\mu\|_{\infty}$.

Theorem 8. Suppose that $\mu$ is a nonzero extremal Beltrami coefficient in $M(R)$. The geodesic segment joining the Teichmüller class of $\mu$ to the basepoint is unique if and only if $\mu$ has constant absolute value and $\left[\mu \chi_{E}+\frac{1}{1+r} \mu \chi_{R-E}\right]$ is a Strebel point for every $r>0$ and every compact subset $E$ of $R$ with positive measure.

\section{The Equivalence Theorem and holomorphic motions of the plane}

Let $\Omega$ be a plane domain and denote by $\Lambda$ its complement in the extended complex plane. A holomorphic motion $h_{t}(z)$ of points $z$ of $\Lambda$ is a mapping $h_{t}$ defined for each $t$ in the unit disc $\Delta$ with the following properties:

$h_{0}$ is the identity mapping,

$h_{t}$ is an injection for all $t \in \Delta$, and

$h_{t}(z)$ is a holomorphic function of $t$ for each fixed $z$ in $\Lambda$.

It is convenient to assume that three points are fixed by the motion $h_{t}$ and, for simplicity, we assume that these three points are 0,1 , and $\infty$. These assumptions are harmless because we can always find a holomorphically varying curve of Möbius transformations $M_{t}$ for which $M_{t} \circ h_{t}(p)=p$ if $p$ is any one of these three points. The parameter $t$ is a called the complex time parameter for the motion.

By a result of Slodkowski ([S1]), any holomorphic motion of $\Lambda$ can be extended to a holomorphic motion of the extended complex plane. This extension is not necessarily unique. For instance, the identity mappings and the vertical stretchings are two different extensions of the holomorphic motion $h_{t}(z)=z$ of the complement of the upper half plane. The extended injections $h_{t}$ are quasiconformal mappings whose Beltrami coefficients depend holomorphically on $t$. On the other hand, any Beltrami coefficient $\mu$ in $M(\Omega)$ defines a holomorphic motion $h_{t}$ of $\Lambda$ by letting $h_{t}(z)$ be the restriction to $\Lambda$ of a quasiconformal homeomorphism of the extended complex plane which fixes 0,1 , and $\infty$, and has Beltrami coefficient $t\left(\mu /\|\mu\|_{\infty}\right) \chi_{\Omega}$. We say that $h_{t}(z)$ so defined is a canonical holomorphic motion generated by the Beltrami coefficient $\mu$. Moreover, by a result of Lieb ([Li]), any holomorphic motion $h_{t}$ of $\Lambda$ is parametrized by a unique basepoint preserving holomorphic mapping $H: \Delta \rightarrow T(\Lambda)=T(\Omega) \times M(\Lambda)$ with $h_{t}(z)=\pi(H(t), z)$, where $\pi([f], z)=f(z)$. This result holds even if $\Omega$ is disconnected (i.e., $\Lambda$ is any closed set), and the notion of equivalence in $T(\Omega)$, where $\Omega$ is a subset of the extended complex plane, might 
be different from the notion of equivalence on the surface which is the union of components of $\Omega$ (see [Li] for more details).

If a canonical holomorphic motion $h_{t}$ is generated by a uniquely extremal Beltrami coefficient $\mu$, then a Beltrami coefficient $\nu_{t}$ of any extension $\tilde{h}_{t}$ of $h_{t}$ to the extended complex plane is equivalent to $t \mu /\|\mu\|_{\infty}$ in $T(\Omega)$. The quasiconformal homeomorphism $\tilde{h}_{t}(z)$ has dilatation less than or equal to $(1+t) /(1-t)$ (see $[\mathrm{BR}]$ ); thus

$$
\left\|\nu_{t}\right\|_{\infty} \leq t
$$

By Theorem $6, t \mu /\|\mu\|_{\infty}$ is uniquely extremal in its class in $T(\Omega)$; thus

$$
\nu_{t}=t \frac{\mu}{\|\mu\|_{\infty}}
$$

Hence we have the following theorem.

Theorem 9. Suppose $h_{t}$ is a canonical holomorphic motion of a closed set $\Lambda$ induced by a Beltrami coefficient $\mu$ which is uniquely extremal with respect to the equivalence relation which defines the Teichmüller space $T(\Omega)$, where $\Omega$ is the complement in the extended complex plane of $\Lambda$. Then $h_{t}$ has a unique extension to a holomorphic motion of the extended complex plane.

Remark. Note that if $\mu$ has constant absolute value, then Theorem 9 was proved by Earle, $\mathrm{Kra}$ and Krushkal, and its proof is an immediate consequence of the condition (c) that we mentioned in Section 8. However, in the next section we show that there are uniquely extremal Beltrami coefficients with non-constant absolute value.

Corollary 1. If $\Lambda=\left\{z: x \leq|y|^{\alpha}\right\}$, with $\alpha \geq 3$, then the affine motion

$$
h_{t}(z)=\frac{1+t}{1-t} x+i y
$$

of $\Lambda$ has a unique extension to the extended complex plane.

Proof. $h_{t}$ is uniquely extremal (see [RS2]) and $h_{t}$ is a canonical motion induced by the Beltrami coefficient of $h_{t}$. Thus, Corollary 1 is an immediate consequence of Theorem 9.

Let $E$ be a Mergelyan subset of a plane domain $\Omega$, that is, a compact subset of $\Omega$ with empty interior and connected complement. Mergelyan sets may have positive measure, and in that case they have an important role in studying the unique extremality (see Lemma 3.1 in [R3]). The key is in the unique extendibility of the vector fields defined on the complement of a Mergelyan set. Suppose that a 
Beltrami coefficient $\mu$ is infinitesimally trivial (i.e., $[\mu]=[0]$ in $B(\Omega)$ ) and vanishes on $\Omega-E$. If $\Omega$ is of infinite analytic type, we may assume with no loss of generality that $C-\Omega$ has a cluster point. Let $V$ be a tangent vector field at $t=0$ to a canonical holomorphic motion of $\Lambda=\Omega^{c}$ generated by $\mu$ (see [EGL]). Then $V$ is a holomorphic function on $C-E$ and vanishes on a set that has a cluster point; thus $V$ vanishes on $E^{c}$. Since $V$ is continuous and $E$ has no interior points, $V(z)=0$ for all $z \in C$. Therefore, $\mu=\bar{\partial} V$ vanishes on $E$. This shows that if $\mu$ and $\nu$ are two infinitesimally equivalent Beltrami coefficients on $\Omega$, and $\mu(z)=\nu(z)$ for all $z$ outside some Mergelyan subset $E$ of $\Omega$, then $\mu \equiv \nu$ (see the treatment of this argument in [R3]).

Lemma 5. Let $\Omega$ be a plane domain of infinite analytic type. If $U$ is an open set in the extended complex plane such that $\bar{U} \subset \Omega$ and $C-\bar{U}$ is connected and dense in $C-U$, then the restrictions to $U$ of quadratic differentials in $A(\Omega)$ are dense in $A(U)$.

Proof. Since $\Omega$ is of infinite analytic type, we may assume with no loss of generality that $C-\Omega$ has a cluster point. Let $A$ be the space of all quadratic differentials in $A(U)$ which are obtained by taking the restrictions of quadratic differentials in $A(\Omega)$. If $A$ is not dense in $A(U)$, then by the Hahn-Banach theorem, there exists a bounded linear functional $L$ on $A(U)$ and a quadratic differential $\varphi$ in $A(U)$ such that $L(\varphi)>0$ and $L(\psi)=0$ for all $\psi \in A$. By the Riesz representation theorem, there exists a Beltrami coefficient $\mu$ on $U$ so that $L(q)=\int_{U} \mu q$, for all $q \in A(U)$. Let $V(z)=L\left(\varphi_{z}\right)$ where

$$
\varphi_{z}(w)=-\frac{z(z-1)}{\pi w(w-1)(w-z)}
$$

That is, $V$ is the initial tangent vector to the normalized holomorphic motion generated by the Beltrami coefficients $t \mu,|t|<1$. We have $\varphi_{z} \in A(U)$ for all $z \in C-U$, and $\varphi_{z} \in A$ for all $z \in C-\Omega$; and, since $L$ vanishes on $A, V$ vanishes on $C-\Omega$. Hence $V$ is holomorphic on the connected set $C-\bar{U}$ and vanishes on a subset that has an accumulation point; thus $V$ vanishes on $C-\bar{U}$. Therefore, $L$ vanishes on the space $R a t$ of all integrable rational functions in the complex plane with all poles located in $C-\bar{U}$. By the Bers' approximation theorem ([A], [B]), Rat is dense in $A(U)$. Thus $L$ vanishes on $A(U)$, a contradiction. 


\section{Uniquely extremal differentials with non-constant modulus}

In this section we assume that $R$ is a Riemann surface which is a subset of another Riemann surface $W$ such that $W-R$ has a cluster point. In particular, $R$ may be any Riemann surface whose universal covering group is of the second kind, or any surface of finite genus and infinite analytic type. We are going to construct uniquely extremal Beltrami coefficients in $T(R)$ which are not constant in absolute value. First, we generalize the ideas from the holomorphic motions in Section 9 to the Riemann surface $R$. We say that a subset $E$ of $R$ is a Mergelyan set, if $E$ is a compact subset of $R$ with empty interior and connected complement.

Lemma 6. If a Beltrami differential $\mu$ is infinitesimally trivial and supported on a Mergelyan set $E$, then $\mu \equiv 0$.

Proof. Fix three points $p_{1}, p_{2}$ and $p_{3}$ in $W-R$ and let $W_{1}=W-\left\{p_{1}, p_{2}, p_{3}\right\}$. Let $H$ be the upper half plane, and $\pi: H \mapsto W_{1}$ the universal covering map with covering group $\Gamma$. Let $B=\pi^{-1}(E), U=\pi^{-1}(R)$, and $\Theta: A(U) \mapsto A(U, \Gamma)=A(R)$ be the Poincaré theta series. Suppose that there exists a nonzero infinitesimally trivial Beltrami differential $\mu \in M(R)$ supported on the set $E$. Lift $\mu$ to the $\Gamma$ automorphic Beltrami differential $\tilde{\mu}$ on $H$,

$$
\tilde{\mu}(z)=\mu(\dot{\pi}(z)) \frac{\overline{\pi^{\prime}(z)}}{\pi^{\prime}(z)} .
$$

Let $V$ be the initial tangent vector to the canonical holomorphic motion generated by $\tilde{\mu}$, that is,

$$
V(z)=\frac{1}{\|\mu\|_{\infty}} \iint_{B} \varphi_{z}(w) \tilde{\mu}(w) d u d v
$$

where

$$
\varphi_{z}(w)=-\frac{1}{\pi} \frac{z(z-1)}{w(w-1)(w-z)} .
$$

Hence, for all $z \in C-U$,

$$
V(z)=\frac{1}{\|\mu\|_{\infty}} \iint_{E} \Theta\left(\varphi_{z}\right) \mu=0 .
$$

Therefore, $V$ is a holomorphic function on $C-B$ and $V(z)=0$ for all $z$ in $C-U$. Since $R-E$ is connected, the restriction of $V$ to any connected component of $C-B$ vanishes on a subset containing a cluster point. Thus $V(z)=0$ for all $z \in C-B$, and since $V$ is continuous and $B$ has no interior points, $V$ vanishes on $H$. 
In the case when $R$ is the unit disc, Lemma 6 was proved by Reich in [R3].

Now we generalize Lemma 5.

Lemma 7. Let $N$ be an open subset of $R$ such that the closure $\bar{N}$ of $N$ is compact and $R-\bar{N}$ is connected and dense in $R-N$. Then the set of restrictions to $N$ of quadratic differentials in $A(R)$ is dense in $A(N)$.

Proof. Let $\pi: H \mapsto W_{1}$ be the universal covering map from Lemma 6 and let $U=\pi^{-1}(R)$ and $D=\pi^{-1}(N)$. Let $A$ be the set of all vectors in $A(N)$ obtained by taking restrictions of quadratic differentials in $A(R)$. If $A$ is not dense in $A(N)$, there exists a linear functional $L$ in $A(N)^{*}$ represented by a Beltrami differential $\mu$ in $M(N)$ and a vector $\varphi \in A(N)$ such that $L(q)=\int_{N} \mu q$ for all $q \in A(N)$, $L(\varphi)>0$, and $L(\psi)=0$ for all $\psi \in A$. Lift $\mu$ to a Beltrami differential $\tilde{\mu} \in M(D)$. Again, let $V(z)=\int_{D} \varphi_{z} \tilde{\mu}$. Then $V(z)=L\left(\Theta\left(\varphi_{z}\right)\right)=0$ for all $z$ in $C-U$, and $V$ is holomorphic on $C-\bar{D}$. Since $W-R$ has a cluster point and $R-N$ is connected, every connected component of $C-\bar{D}$ contains a cluster point of $C-U$; thus $V$ vanishes on $C-\bar{D}$. By the Bers' approximation theorem, the linear span of quadratic differentials $\varphi_{z}$ over all $z$ in $C-\bar{D}$ is dense in $A(D)$. Since the Poincare series $\Theta_{D}: A(D) \rightarrow A(D, \Gamma)=A(N)$ is surjective (see [Kr]), $L$ vanishes on $A(N)$, a contradiction.

Lemmas 6 and 7 are consequences of Bers' approximation theorem. In case $R$ is a bounded plane domain, they are also consequences of Mergelyan's theorem. Reich suggested that if uniquely extremal Beltrami coefficients with nonconstant modulus exist, Mergelyan's theorem might play a role. Next, we show that suitable generalizations in Lemmas 6 and 7, together with the generalized Infinitesimal Delta Inequality from Section 6, indeed play a role in constructing uniquely extremal Beltrami coefficients with non-constant absolute value.

Theorem 10 (Counterexample Theorem). Let $R$ be a Riemann surface which is a subset of another Riemann surface $W$ such that $W-R$ has a cluster point. Then there is a uniquely extremal Beltrami coefficient $\mu$ on $R$ such that $|\mu|$ is not a.e. constant on $R$.

Proof. Fix a unit vector $q$ in $A(R)$. We define the $q$-measure of a Borel subset $S$ of $R$ by $\int_{S}|q(z)| d x d y$. Take a Mergelyan subset $E$ of $R$ with positive measure and pick two sequences $U_{n}$ and $V_{n}$ of open sets in $R$ such that $E \subset \overline{U_{n+1}} \subset$ $U_{n}, \overline{V_{n-1}} \subset V_{n}, \overline{U_{n}} \cap \overline{V_{n}}=\emptyset$, the $q$-measure of $R-\left(\bigcup_{n=1}^{\infty} V_{n} \cup E\right)$ is zero, and $R-\left(\overline{U_{n}} \cup \overline{V_{n}}\right)$ is connected and dense in $R-\left(U_{n} \cup V_{n}\right)$. To construct a uniquely extremal Beltrami differential $\mu$ with nonconstant modulus, we let $\mu$ equal zero on $E$. Outside $E, \mu$ is defined as the limit of $\left|\varphi_{n}\right| / \varphi_{n}$, where the sequence $\varphi_{n} \in A(R)$ is 
chosen to satisfy Reich's condition, that is, $\delta\left(\varphi_{n}\right) \rightarrow 0$ and $\liminf _{n \rightarrow \infty}\left|\varphi_{n}(z)\right|>0$ for almost all $z$ in $R-E$. If $\mu$ is not uniquely extremal in its class in $B(R)$, then by Lemma 6 , there exists a Beltrami coefficient $\nu$ infinitesimally equivalent to $\mu$ and different from $\mu$ on the set $E^{c}$. Thus, there exists $\epsilon>0$ and a compact subset $G$ of $E^{c}$ with positive measure such that $|\mu(z)-\nu(z)|>\epsilon$ for almost all $z$ in $G$. By the generalized Infinitesimal Delta Inequality (Theorem 4) and Fatou's lemma, $0<\epsilon^{2} \int_{G} \lim \inf \left|\varphi_{n}\right| \leq C \lim \inf \delta\left(\varphi_{n}\right)$, a contradiction. Thus $\mu$ is uniquely extremal in its class in $B$; hence by the Equivalence Theorem, $\mu$ is uniquely extremal in its Teichmüller class.

Now we start with the construction of the sequence $\varphi_{n}$. Let $q_{1} \in A\left(U_{2} \cup V_{2}\right)$ be defined as $q_{1}(z)=0$ for all $z$ in $U_{2}$, and $q_{1}(z)=2 q(z)$ for all $z$ in $V_{2}$. By Lemma 7 , there exists a sequence of non-zero vectors $\psi_{n}$ in $A(R)$ such that

$$
\left\|\psi_{n}-q_{1}\right\|_{U_{2} \cup V_{2}} \rightarrow 0 .
$$

Take a compact subset $W_{0}$ of $V_{2}$ such that the interior of $W_{0}$ contains all zeros of $q$ in $\overline{V_{1}}$ and $\int_{W_{0}}\left|q_{1}\right| \leq 1 / 2$. Since $\psi_{n}$ converges to $q_{1}$ uniformly on $E \cup \overline{V_{1}}$, there is a sufficiently large integer $N$ such that $\int_{E}\left|\psi_{N}(z)\right| \leq 1 / 8$ and $\left|\psi_{N}(z)\right| \geq|q(z)|$ for all $z \in \overline{V_{1}}-W_{0}$. Let $\varphi_{1}=\psi_{N}$. Since the $q$-measure of $R-\left(\bigcup_{n=1}^{\infty} V_{n} \cup E\right)$ is zero, we may pick $V_{k_{1}}$ such that $\int_{R-V_{k_{1}}}\left|\varphi_{1}\right| \leq 1 / 4$. Take a compact subset $W_{1}$ of $V_{k_{1}+1}$ such that the interior of $W_{1}$ contains all zeros of $\varphi_{1}$ in $\overline{V_{k_{1}}}, \int_{W_{1}}\left|\varphi_{1}\right| \leq 1 / 4$, and the $q$-measure of $W_{1}$ is less than $1 / 2$. Let $q_{2} \equiv 0$ on $U_{k_{1}+1}$ and $q_{2} \equiv 2 \varphi_{1}$ on $V_{k_{1}+1}$. Then $q_{2} \in A\left(U_{k_{1}+1} \cup V_{k_{1}+1}\right)$; and, by Lemma 7, there exists a sequence of non-zero vectors $a_{n}$ in $A(R)$ such that $\left\|a_{n}-q_{2}\right\|_{U_{k_{1}+1} \cup V_{k_{1}+1}} \rightarrow 0$. Therefore, $a_{n}$ converges to $q_{2}$ uniformly on $E \cup \overline{V_{k_{1}}}$. Thus there is a sufficiently large integer $M$, such that the following three conditions hold:

(i) $\int_{E}\left|a_{M}(z)\right| \leq 1 / 8$,

(ii) $\left|a_{M}(z)\right| \geq\left|\varphi_{1}(z)\right|$ for all $z \in V_{k_{1}}-W_{1}$, and

(iii) $\left|\frac{\left|a_{M}(z)\right|}{a_{M}(z)}-\frac{\left|\varphi_{1}(z)\right|}{\varphi_{1}(z)}\right| \leq \frac{1}{2 \int_{V_{k_{1}}}\left|\varphi_{1}\right|}$ for all $z \in V_{k_{1}}-W_{1}$.

Let $\varphi_{2}=a_{M}$. By repeating this construction, we obtain a sequence of non-zero quadratic differentials $\varphi_{n}$ in $A(R)$, a subsequence $V_{k_{n}}$ of $V_{k}$, and a sequence of compact subsets $W_{n}$ of $V_{k_{n}+1}$ which satisfy the following conditions.

(1) $\int_{E}\left|\varphi_{n+1}(z)\right| \leq 1 / 2^{n+2}$,

(2) $\left|\varphi_{n+1}(z)\right| \geq\left|\varphi_{n}(z)\right|$ for all $z$ in $V_{k_{n}}-W_{n}$,

(3) the $q$-measure of $W_{n+1}$ is less than $1 / 2^{n+1}$, 
(4) $W_{n+1}$ contains all zeros of $\varphi_{n+1}$ in $\overline{V_{k_{n+1}}}$,

(5) $\int_{W_{n+1}}\left|\varphi_{n+1}\right| \leq 1 / 2^{n+2}$,

(6) $\left|\frac{\left|\varphi_{n+1}(z)\right|}{\varphi_{n+1}(z)}-\frac{\left|\varphi_{n}(z)\right|}{\varphi_{n}(z)}\right| \leq \frac{1}{2^{n} \int_{V_{k_{n}}}\left|\varphi_{n}\right|}$ for all $z \in V_{k_{n}}-W_{n}$,

(7) $V_{k_{n}}-W_{n} \subset V_{k_{n+1}}-W_{n+1}$, and

(8) $\int_{R-V_{K_{n+1}}}\left|\varphi_{n+1}\right| \leq 1 / 2^{n+2}$.

To see how the induction works, suppose that conditions (1) through (8) hold at level $n$. Let $q_{n+2} \equiv 0$ on $U_{k_{n+1}+1}$ and $q_{n+2} \equiv 2 \varphi_{n+1}$ on $V_{k_{n+1}+1}$. Then $q_{n+2} \in$ $A\left(U_{k_{n+1}+1} \cup V_{k_{n+1}+1}\right)$, and by Lemma 7 , there exists a sequence of non-zero vectors $a_{m}$ in $A(R)$ such that $\left\|a_{m}-q_{n+2}\right\|_{U_{k_{n+1}+1} \cup V_{k_{n+1}+1}} \rightarrow 0$. By letting $\varphi_{n+2}=a_{M}$ for a sufficiently large integer $M$, we get conditions (1), (2) and (6) on the next inductive level (with $n$ replaced by $n+1$ ). Since $R-\left(\bigcup_{m=1}^{\infty} V_{m} \cup E\right.$ ) has measure zero, we may pick $V_{k_{n+2}}$ such that the next level of condition (8) holds. Finally, we take a compact subset $W_{n+2}$ of $V_{k_{n+2}+1}$ such that the next $(n+1)$ level of conditions (3), (4), (5) and (7) hold.

By conditions (2), (6), and (7), the sequence $\left|\varphi_{n}(z)\right| / \varphi_{n}(z)$ is a Cauchy sequence in each $L^{\infty}\left(V_{k_{j}}-W_{j}\right)$. Hence $\left|\varphi_{n}(z)\right| / \varphi_{n}(z)$ converges to some $\mu(z)$ for all $z$ in $\bigcup_{j=1}^{\infty}\left(V_{k_{j}}-W_{j}\right)$, and $\mu(z)=0$ for all $z$ in $E$. By (3), $\mu(z)$ is defined for almost all $z$ in $R$, and $|\mu(z)|=1$ for almost all $z$ in $R-E$. Furthermore,

$$
\begin{gathered}
0 \leq \delta\left(\varphi_{n}\right)=\int_{R}\left|\varphi_{n}\right|-\operatorname{Re} \int_{R} \mu \varphi_{n} \\
\leq \int_{V_{k_{n}}-W_{n}}\left|\varphi_{n}\right|-\operatorname{Re} \int_{V_{k_{n}}-W_{n}} \mu \varphi_{n}+2 \int_{R-V_{k_{n}}}\left|\varphi_{n}\right|+2 \int_{W_{n}}\left|\varphi_{n}\right| .
\end{gathered}
$$

Hence, by (5) and (8),

$$
\delta\left(\varphi_{n}\right) \leq \int_{V_{k_{n}}-W_{n}}\left|\varphi_{n}\right|-\operatorname{Re} \int_{V_{k_{n}}-W_{n}} \mu \varphi_{n}+\frac{1}{2^{n-1}}
$$


Furthermore, (2), (6), and (7) yield

$$
\begin{gathered}
\int_{V_{k_{n}}-W_{n}}\left|\varphi_{n}\right|-\operatorname{Re} \int_{V_{k_{n}}-W_{n}} \mu \varphi_{n} \leq \int_{V_{k_{n}-W_{n}}}\left|\varphi_{n}\right|\left|\mu-\frac{\left|\varphi_{n}\right|}{\varphi_{n}}\right| \\
=\lim _{m \rightarrow \infty} \int_{V_{k_{n}}-W_{n}}\left|\varphi_{n}\right|\left|\frac{\left|\varphi_{m}\right|}{\varphi_{m}}-\frac{\left|\varphi_{n}\right|}{\varphi_{n}}\right| \leq \lim _{m \rightarrow \infty} \sum_{j=n}^{m-1} \int_{V_{k_{n}}-W_{n}}\left|\varphi_{n}\right|\left|\frac{\left|\varphi_{j+1}\right|}{\varphi_{j+1}}-\frac{\left|\varphi_{j}\right|}{\varphi_{j}}\right| \\
\leq \lim _{m \rightarrow \infty} \sum_{j=n}^{m-1} \int_{V_{k_{n}}-W_{n}}\left|\varphi_{j}\right|\left|\frac{\left|\varphi_{j+1}\right|}{\varphi_{j+1}}-\frac{\left|\varphi_{j}\right|}{\varphi_{j}}\right| \leq \lim _{m \rightarrow \infty} \sum_{j=n}^{m-1} \int_{V_{k_{j}}-W_{j}}\left|\varphi_{j}\right|\left|\frac{\left|\varphi_{j+1}\right|}{\varphi_{j+1}}-\frac{\left|\varphi_{j}\right|}{\varphi_{j}}\right| \\
\leq \lim _{m \rightarrow \infty} \sum_{j=n}^{m-1} \frac{1}{2^{j}} \leq \frac{1}{2^{n-1}} .
\end{gathered}
$$

Theorem 11. Let $R$ be a Riemann surface which is a subset of another Riemann surface $W$ such that $W-R$ has a cluster point, and let $q$ be a unit vector in $A(R)$. Then for every $\epsilon>0$ and every Beltrami differential $\mu$ in $M(R)$, there exists a uniquely extremal Beltrami coefficient $\nu$ on $R$ such that the q-measure of the set where $\mu$ is different from $\nu$ is less than $\epsilon$.

Proof. Let $k=\|\mu\|_{\infty}$. Choose a Mergelyan subset $E$ of $R$ such that the $q$ measure of $R-E$ is less than $\epsilon$. By imitating the proof of the Counterexample Theorem, construct a uniquely extremal Beltrami coefficient $\eta$ on $R$ such that $\|\eta\|_{\infty}>k$ and $\eta(z)=0$ for all $z \in E$. We leave to the reader to check that this construction is easily effected in the case when $R$ is the unit disc or an annulus. In the case when $R$ is more complicated, take a simple closed Poincaré geodesic $\gamma$ on $R$. Then there exists a Jenkins-Strebel differential $\varphi \in A(R)$ whose only cylinder $c$ has a core curve freely homotopic to $\gamma$, and the measure of $R-c$ is zero (see [S5] for more details). Thus the construction reduces to the case of an annulus. (Another approach to this construction may be obtained by using a fundamental domain of $R$.) Define $\nu \in M(R)$ by $\nu(z)=\eta(z)$ for all $z \in R-E$, and $\nu(z)=\mu(z)$ for $z \in E$. Then by Step II in the proof of Lemma $4, \nu(z)$ is uniquely extremal.

\section{Characterizations of unique extremality}

Let $R$ be a Riemann surface whose universal covering surface is the unit disc. If $\mu \in L^{\infty}(R)$, we say that the set $X(\mu)=\left\{z \in R:|\mu(z)|=\|\mu\|_{\infty}\right\}$ is the extremal set for the Beltrami differential $\mu$. The extremal sets play the fundamental role in 
our characterizations of unique extremality. As an illustration, we start with the following lemma.

Lemma 8. Let $\mu \in L^{\infty}(R)$ be a uniquely extremal Beltrami differential and let $E=\{z \in R:|\mu(z)| \leq s\}$ where $s<\|\mu\|_{\infty}$. Then the space of all restrictions to $E$ of quadratic differentials in $A(R)$ is dense in $L^{1}(E)$.

Proof. Suppose that $A$ is not dense in $L^{1}(E)$. Then by the Hahn-Banach theorem there exists a non-trivial bounded linear functional $L$ on $L^{1}(E)$ which vanishes on $A(R)$. By the Riesz representation theorem, there exists a nonzero $\nu \in L^{\infty}(R)$ supported on $E$ such that $0=L(\varphi)=\int_{E} \nu \varphi$ for all $\varphi \in A(R)$. Thus, for sufficiently small $t>0$, the Beltrami differential $\mu+t \nu$ is infinitesimally equivalent to $\mu$ and satisfies $\|\mu+t \nu\|_{\infty} \leq\|\mu\|_{\infty}$, contradicting the unique extremality of $\mu$.

To obtain a characterization of unique extremality in the general case, we study the proof of the Hahn-Banach theorem. Let $M$ be a subspace of a normed space $X$, and let $\Lambda$ be a bounded linear functional on $M$ and $\lambda$ its real part. Let

$$
\bar{\lambda}\left(x_{0}\right)=\inf _{y \in M}\left\{\lambda(y)+\|\lambda\|\left\|y-x_{0}\right\|\right\}
$$

and

$$
\underline{\lambda}\left(x_{0}\right)=\sup _{x \in M}\left\{\lambda(x)-\|\lambda\|\left\|x-x_{0}\right\|\right\} .
$$

The analysis of the proof of the Hahn-Banach theorem leads to the following lemma.

Lemma 9. $\Lambda$ has a unique norm-preserving extension from $M$ to $X$ if and only if $\bar{\lambda}\left(x_{0}\right)=\underline{\lambda}\left(x_{0}\right)$ for all $x_{0}$ in $X-M$.

We say that $\lambda$ satisfies the unique approximation property at $x_{0} \in X-M$ if there exist sequences $u_{n}$ and $v_{n}$ in $M$ such that

$$
\lambda\left(u_{n}-v_{n}\right)=\|\lambda\|\left(\left\|u_{n}-x_{0}\right\|+\left\|x_{0}-v_{n}\right\|\right)+o(1) .
$$

Recall that the the functional $\delta=\delta_{\lambda}$ is defined on $M$ by

$$
\delta(\varphi)=\|\lambda\|\|\varphi\|-\lambda(\varphi)
$$

We say that a sequence $\varphi_{n}$ in $M$ is a weak Hamilton sequence for $\lambda$ if $\delta_{\lambda}\left(\varphi_{n}\right)$ converges to zero as $n$ tends to infinity.

Lemma 10. If $\lambda$ has a unique norm-preserving extension from $M$ to $X$, then for each $x_{0} \in X-M$, there exist sequences $u_{n}$ and $v_{n}$ in $M$ such that 
(1) $\lambda\left(u_{n}\right)=\lambda\left(x_{0}\right)+\|\lambda\|\left\|u_{n}-x_{0}\right\|+o(1)$,

(2) $\lambda\left(v_{n}\right)=\lambda\left(x_{0}\right)-\|\lambda\|\left\|v_{n}-x_{0}\right\|+o(1)$,

(3) $\lambda\left(u_{n}-v_{n}\right)=\|\lambda\|\left(\left\|x_{0}-u_{n}\right\|+\left\|x_{0}-v_{n}\right\|\right)+o(1)$, and

(4) $u_{n}-v_{n}$ is a weak Hamilton sequence for $\lambda$.

Proof. We may suppose with no loss of generality that $\|\lambda\|=1$. If $\lambda$ has a unique Hahn-Banach extension (which we continue to denote by $\lambda$ ) from $M$ to $X$, then by Lemma 9 , for each $x_{0}$ in $X-M$ there exist sequences $u_{n}$ and $v_{n}$ in $M$ such that

$$
\lambda\left(u_{n}\right)-\left\|u_{n}-x_{0}\right\|+o(1)=\lambda\left(x_{0}\right)=\lambda\left(v_{n}\right)+\left\|v_{n}-x_{0}\right\|+o(1) .
$$

Hence $\lambda\left(w_{n}\right)=\left\|x_{0}-u_{n}\right\|+\left\|x_{0}-v_{n}\right\|+o(1)$, where $w_{n}=u_{n}-v_{n}$. Combining this inequality with

$$
\lambda\left(w_{n}\right) \leq\left\|w_{n}\right\| \leq\left\|x_{0}-u_{n}\right\|+\left\|x_{0}-v_{n}\right\|,
$$

we conclude that

$$
\left\|w_{n}\right\|=\left\|x_{0}-u_{n}\right\|+\left\|x_{0}-v_{n}\right\|+o(1)
$$

and $\lambda\left(w_{n}\right)=\left\|w_{n}\right\|+o(1)$.

The following result is an immediate consequence of the previous two lemmas.

Theorem 12. Let $\Lambda$ be a bounded linear functional on $M$ and $\lambda$ its real part. Then $\Lambda$ has a unique norm-preserving extension from $M$ to $X$ if and only if $\lambda$ satisfies the unique approximation property at each $x_{0}$ in $X-M$.

Following the discussion of Reich's sufficient condition for the unique extremality in Section 5 (see [R1] and [R4] for more details), we say that $\mu \in L^{\infty}(R)$ satisfies Reich's condition on a set $E \subset R$ if there exists a sequence $\varphi_{n}$ in $A(R)$ so that $\delta\left(\varphi_{n}\right) \rightarrow 0$ and $\liminf \left|\varphi_{n}(z)\right|>0$ for almost all $z$ in $E$.

Lemma 11. If $\mu \in L^{\infty}(R)$ is uniquely extremal in its class in $B(R)$, then $\mu$ satisfies Reich's condition on its extremal set.

Proof. Suppose that $\mu$ is uniquely extremal in its class in $B(R)$ and let $E=$ $X(\mu)$ be its extremal set. Without loss of generality, we assume $\|\mu\|_{\infty}=1$. Take a unit vector $q$ in $A(R)$, and let $f=|q| \bar{\mu} \chi_{E}$. Clearly, $f \in L^{1}(R)$ and

$$
\int_{R} f \mu=\int_{E}|q|=\int_{E}|f|=\|f\| .
$$

By condition (1) of Lemma 10, there exists a sequence $\varphi_{n}$ in $A(R)$ such that

$$
\operatorname{Re} \int_{R} \mu f+\left\|f-\varphi_{n}\right\|-\operatorname{Re} \int_{R} \varphi_{n} \mu \rightarrow 0 \text {. }
$$


Hence,

$$
\begin{aligned}
0 & \leq\|f\|+\left\|f-\varphi_{n}\right\|-\left\|\varphi_{n}\right\| \\
& \leq \operatorname{Re} \int_{R} \mu f+\left\|f-\varphi_{n}\right\|-\operatorname{Re} \int_{R} \varphi_{n} \mu \rightarrow 0
\end{aligned}
$$

and

$$
\begin{aligned}
0 & \leq\left\|\varphi_{n}\right\|-\operatorname{Re} \int_{R} \varphi_{n} \mu \\
& \leq\|f\|+\left\|f-\varphi_{n}\right\|-\operatorname{Re} \int_{R} \varphi_{n} \mu \\
& =\operatorname{Re} \int_{R} \mu f+\left\|f-\varphi_{n}\right\|-\operatorname{Re} \int_{R} \varphi_{n} \mu \rightarrow 0 .
\end{aligned}
$$

Thus

$$
\delta\left(\varphi_{n}\right) \rightarrow 0 .
$$

Since $0 \leq \int_{E}\left(|f|+\left|f-\varphi_{n}\right|-\left|\varphi_{n}\right|\right) \leq\|f\|+\left\|f-\varphi_{n}\right\|-\left\|\varphi_{n}\right\| \rightarrow 0$, by passing to a subsequence, we may assume that $|f(z)|+\left|f(z)-\varphi_{n}(z)\right|-\left|\varphi_{n}(z)\right| \rightarrow 0$, for almost all $z \in E$. Hence, $\liminf _{n \rightarrow \infty}\left|\varphi_{n}(z)\right| \geq|f(z)|=|q(z)|>0$ for almost all $z \in E$.

If $\mu$ has constant absolute value, the converse of Lemma 11 holds, and this provides another characterization of the unique extremality of $\mu$ (see Theorem 1).

Theorem 13. If $\mu \in L^{\infty}(R)$ has constant modulus, then $\mu$ is uniquely extremal in its infinitesimal Teichmüller class if and only if $\mu$ satisfies Reich's condition on $R$.

Proof. Lemma 11 shows that if $\mu$ is uniquely extremal and has constant absolute value, then $\mu$ satisfies Reich's condition on $R$. To prove the converse, suppose that $\mu$ has constant absolute value and satisfies Reich's condition on $R$. First we show that $\mu$ is extremal in its infinitesimal class. Since $\mu$ satisfies Reich's condition on $R$, there exists a sequence $\varphi_{n}$ in $A(R)$ such that $\delta\left(\varphi_{n}\right) \rightarrow 0$ and $\lim \inf \left|\varphi_{n}(z)\right|>0$ for almost all $z \in R$. Therefore, by Fatou's lemma,

$$
\liminf _{n \rightarrow \infty}\left\|\varphi_{n}\right\|>0
$$

Hence

$$
\|\mu\|_{\infty}-\operatorname{Re} \int_{R} \mu \frac{\varphi_{n}}{\left\|\varphi_{n}\right\|}=\frac{1}{\left\|\varphi_{n}\right\|} \delta\left(\varphi_{n}\right) \rightarrow 0
$$

thus $\mu$ is extremal.

If $\mu$ is not the unique extremal, then there exists $\nu \in L^{\infty}(R)$ equivalent to $\mu$, such that $|\mu(z)-\nu(z)| \geq \epsilon>0$ for all $z$ in some compact set $F$ of positive measure. 
The generalized Infinitesimal Delta Inequality (Theorem 4) yields

$$
\epsilon^{2} \int_{F}|\varphi| \leq C|| \mu \|_{\infty} \delta(\varphi)
$$

for all $\varphi \in A(R)$. On the other hand, $\mu$ satisfies Reich's condition on $R$. Thus, there exists a sequence $\varphi_{n}$ in $A(R)$ such that $\delta\left(\varphi_{n}\right) \rightarrow 0$ and $\lim \inf \left|\varphi_{n}(z)\right|>0$ for almost all $z \in R$. Applying Fatou's lemma, we obtain the contradiction.

Using Theorem 13, we may now generalize Theorem 1 and give characterizations for the unique extremality of an arbitrary Beltrami differential in $M(R)$. We say that a Beltrami coefficient $\eta$ is an admissible variation of $\mu$ if $\|\eta\|_{\infty} \leq\|\mu\|_{\infty}$ and there exist a subset $P$ of $R$ and a real number $s<\|\mu\|_{\infty}$ such that $|\mu(z)| \leq s$ for almost every $z$ in $P$ and $\mu(z)=\eta(z)$ for almost all $z$ in $R-P$.

Theorem 14 (Characterization Theorem). Let $\mu \in M(R)$. The following conditions are equivalent.

(1) $\mu$ is uniquely extremal in its class in $T$;

(2) $\mu$ is uniquely extremal in its class in $B$;

(3) for every admissible variation $\eta$ of $\mu$ and every compact subset $E$ of $X(\eta)$ with nonzero measure, there exists a sequence of unit vectors $\varphi_{n}$ in $A(R)$ such that

$$
\frac{1}{\int_{E}\left|\varphi_{n}\right|}\left(\|\eta\|_{\infty}-\operatorname{Re} \int_{R} \eta \varphi_{n}\right) \rightarrow 0
$$

(4) for every admissible variation $\eta$ of $\mu$ and every compact subset $E$ of $X(\mu)$ with nonzero measure, there exists a sequence of unit vectors $\varphi_{n}$ in $A(R)$ such that

$$
\frac{1}{\int_{E}\left|\varphi_{n}\right|} \operatorname{Re} \int_{E} \eta \varphi_{n} \rightarrow\|\eta\|_{\infty}
$$

and

$$
\frac{1}{\int_{E}\left|\varphi_{n}\right|}\left(\|\eta\|_{\infty} \int_{R-E}\left|\varphi_{n}\right|-\operatorname{Re} \int_{R-E} \eta \varphi_{n}\right) \rightarrow 0
$$

(5) $\mu$ is extremal in its Teichmüller class and for every $r>0$, every admissible variation $\eta$ of $\mu$, and every compact subset $E$ of $X(\eta)$ with nonzero measure, $\left[\eta \chi_{E}+\frac{1}{1+r} \eta \chi_{R-E}\right]$ is a Strebel point in $T$;

(6) $\mu$ is extremal in its infinitesimal class and for every $r>0$, every admissible variation $\eta$ of $\mu$, and every compact subset $E$ of $X(\eta)$ with nonzero measure, $\left[\eta \chi_{E}+\frac{1}{1+r} \eta \chi_{R-E}\right]$ is an infinitesimal Strebel point in $B(R)$;

(7) every admissible variation $\eta$ of $\mu$ satisfies Reich's condition on the extremal set of $\eta$; and

(8) $\lambda_{\mu}$ satisfies the unique approximation property at each $x_{0} \in L^{1}(R)-A(R)$. 
Proof. (1) is equivalent to (2) by the Equivalence Theorem. (2) and (8) are equivalent by Theorem 12. Clearly, (3) is equivalent to (4) by the same reasoning used to prove (c) is equivalent to (d) in Theorem 1. If $\mu$ is any uniquely extremal Beltrami coefficient, so is every admissible variation $\eta$ of $\mu$, by Step II in the proof of the Equivalence Theorem. Thus, by Lemma 11, (2) implies (7). Again, if $\mu$ satisfies (2), so does any admissible variation $\eta$ of $\mu$; thus by the proof of Lemma 2, (2) implies (6). The parallel reasoning (i.e., using the proof of Lemma 1) shows that (1) implies (5). The calculation in the last paragraph of the proof of Lemma 1 shows that (5) implies (3), and the last paragraph of the proof of Lemma 2 shows that (6) implies (3). From the generalized Infinitesimal Delta Inequality, we conclude that (3) or (7) implies that every admissible variation $\eta$ of $\mu$ is uniquely extremal on its extremal set $X(\eta)$; thus by Step III in the proof of the Equivalence Theorem, (3) implies (2) and (7) implies (2).

In the Counterexample Theorem we showed that the complement of the extremal set of a uniquely extremal Beltrami differential may have positive measure. In our final theorem we use the Characterization Theorem to show there are uniquely extremal Beltrami differentials whose extremal sets are empty.

Theorem 15. Let $R$ be a Riemann surface which is a subset of another Riemann surface $W$ and suppose that the complement of $R$ in $W$ has a cluster point. Then there exists a uniquely extremal Beltrami coefficient $\mu$ on $R$ whose extremal set $X(\mu)$ is empty.

Proof. Let $f_{n}$ be a sequence in $L^{1}(R)$ such that each member of the sequence appears infinitely many times and which is dense in $L^{1}(R)$. Inductively, we define a sequence of uniquely extremal Beltrami coefficients $\mu_{n}$ on $R$ and two sequences $u_{n}$ and $v_{n}$ in $A(R)$, such that

(1) $\left\|\mu_{n}\right\|_{\infty}<\left\|\mu_{n+1}\right\|_{\infty}<1$, and

(2) $\left\|\lambda_{n}\right\|\left(\left\|u_{i}-f_{i}\right\|+\| v_{i}-f_{i} \mid\right)-\lambda_{n}\left(u_{i}-v_{i}\right)<2^{-i}$, for each $1 \leq i \leq n$, where $\lambda_{n}$ is the linear functional on $A(R)$ defined by $\lambda_{n}(\varphi)=\operatorname{Re} \int_{R} \varphi \mu_{n}$.

Note that $\left\|\lambda_{n}\right\|=\left\|\mu_{n}\right\|_{\infty}$. Suppose that we have already found $\mu_{i}, u_{i}$ and $v_{i}$, $1 \leq i \leq N$. Let $E_{1} \subset E_{2} \subset E_{3} \subset \cdots$ be an exhaustion of $R$ (up to a set of measure zero) by Mergelyan sets (see the proof of Corollary 2 for the construction of the sequence $E_{n}$ ).

By Theorem 11 and Step II in Lemma 4, we may choose a uniquely extremal Beltrami differential $\mu_{N+1}$ such that $\left\|\mu_{N}\right\|_{\infty}<\left\|\mu_{N+1}\right\|_{\infty}<1$, and $\mu_{N}=\mu_{N+1}$ on some $E_{m}$. In addition, if we choose $m$ large enough, we can force $\mu_{N+1}$ to satisfy inequality (2) for each $1 \leq i \leq N$. Then, since $\mu_{N+1}$ is uniquely extremal, we can 
choose quadratic differentials $u_{N+1}$ and $v_{N+1}$ such that inequality (2) holds for $n=N+1$ and $1 \leq i \leq n$.

Hence, there exists a differential $\mu \in L^{\infty}(R)$ such that $\mu_{n} \rightarrow \mu$ a.e. on $R$ and $|\mu(z)|<\|\mu\|_{\infty} \leq 1$ for almost all $z$. Therefore, $\lambda_{n}(\varphi) \rightarrow \lambda_{\mu}(\varphi)$ for all $\varphi$ in $A(R)$. Thus inequality (2) holds for $\lambda$ and each $i$.

Since for each $f_{m}$, there exists a subsequence $f_{n_{k}}$ of $f_{n}$ such that $f_{m}=f_{n_{k}}$ for each $k$, we conclude that $\lambda_{\mu}$ satisfies the unique approximation property at each $f_{m}$. Hence, since the set of all $f_{n}$ is dense in $L^{1}(R)$, we conclude that $\lambda_{\mu}$ satisfies the unique approximation property at each $f \in L^{1}(R)$. Thus, by the Characterization Theorem and the Equivalence Theorem, $\frac{1}{2} \mu$ is uniquely extremal in its Teichmüller class.

\section{Acknowledgments}

We would like to thank Clifford J. Earle, Frederick P. Gardiner, Edgar Reich and Kurt Strebel for motivation and many helpful discussions and suggestions concerning our research and related literature.

\section{REFERENCES}

[A] L. Ahlfors, Finitely generated Kleinian groups, Amer. J. Math. 86 (1964), 413-429; 87 (1965), 759.

[B] L. Bers, An approximation theorem, J. Analyse Math. 14 (1965) 1-4.

[BA] A. Beurling and L. Ahlfors, The boundary correspondence under quasiconformal mappings, Acta Math. 96 (1956), 125-142.

[BR] L. Bers and H. L. Royden, Holomorphic families of injections, Acta Math. 157 (1986), 259-286.

[EKK] C. J. Earle, I. Kra and S. L. Krushkal, Holomorphic motions and Teichmüller spaces, Trans. Amer. Math. Soc. 343 (1994), 927-948.

[EG] C. J. Earle and F. P. Gardiner, Geometric isomorphisms between infinite dimensional Teichmüller spaces, Trans. Amer. Math. Soc. 348 (1996), 1163-1190.

[EGL] C. J. Earle, F. P. Gardiner and N. Lakic, Vector fields for holomorphic motions of closed sets, Contemp. Math. 211 (1997), 193-225.

[ELa] C. J. Earle and N. Lakic Variability set on a Riemann surface, preprint.

[EL] C. J. Earle and Zhong Li, Isometrically embedded polydisks in infinite dimensional Teichmüller spaces, J. Geom. Anal., to appear.

[EL2] C. J. Earle and Zhong Li, Extremal quasiconformal mappings, preprint.

[G] F. P. Gardiner, Teichmüller Theory and Quadratic Differentials, Wiley-Interscience, New York, 1987. 
[Gr] H. Grötzsch, Über die Verzerrung bei schlichten nicht konformen Abbildungen und über eine damit zusammenhängende Erweiterung des Picardschen Satzes, Ber. Verh. Sächs. Akad. Wiss. Leipzig 80 (1928), 503-507.

[H] R. S. Hamilton, Extremal quasiconformal mappings with prescribed boundary values, Trans. Amer. Math. Soc. 138 (1969), $399-406$.

[K] S. Krushkal, Extremal quasiconformal mappings, Siberian Math. J. 10 (1969), 411-418.

[Kr] I. Kra, Automorphic Forms and Kleinian Groups, Benjamin, Reading, Massachusetts, 1972.

[L1] N. Lakic, Strebel points, Contemp. Math. 211 (1997), 417-431.

[L2] N. Lakic, Infinitesimal Teichmüller geometry, Complex Variables Theory Appl. 30 (1996), 1-17.

[Li] G. S. Lieb, Holomorphic motions and Teichmüller space, Ph.D. Thesis, Cornell Univ., 1990.

[Liz] Zhong Li, Nonuniqueness of geodesics in infinite dimensional Teichmüller spaces, Complex Variables Theory Appl. 16 (1991), 261-272.

[MM] M. Mateljević and V. Marković, The unique extremal q.c. mappings and uniqueness of HahnBanach extension, Matematichki Vesnik 48 (1996), 107-112.

[P] M. Pavlovic, in preparation.

[R1] E. Reich, On criteria for unique extremality of Teichmüller mappings, Ann. Acad. Sci. Fenn. Ser. A I Math. 6 (1981), 289-301.

[R2] E. Reich, Extremal extensions from the circle to the disk, preprint.

[R3] E. Reich, On the uniqueness question for Hahn-Banach extensions from the space of $L^{1}$ analytic functions, Proc. Amer. Math. Soc. 88 (1983), 305-310.

[R4] E. Reich, On criteria for unique extremality of Teichmüller mappings, Indiana Univ. Math. J. 30 (1981), 441-447.

[R5] E. Reich, An extremum problem for analytic functions with area norm, Ann. Acad. Sci. Fenn. Ser. A I Math. 2 (1976), 429-445.

[RS] E. Reich and K. Strebel, Extremal quasiconformal mappings with given boundary values, in Contributions to Analysis (L. Ahlfors et al., eds.), Academic Press, New York, 1974, pp. 375392.

[RS2] E. Reich and K. Strebel, On the extremality of certain Teichmüller mappings, Comment. Math. Helv. 45 (1970), 353-362.

[S1] Z. Slodkowski, Holomorphic motions and polynomial hulls, Proc. Amer. Math. Soc. 111 (1991), 347-355.

[S1] K. Strebel, On quasiconformal mappings of open Riemann surfaces, Comment. Math. Helv. 53 (1978), 301-321.

[S2] K. Strebel, On the existence of extremal Teichmüller mappings, J. Analyse Math. 30 (1976), $464-480$.

[S3] K. Strebel, Eine abschätzung der länge gewisser kurven bei quasikonformer Abbildung, Ann. Acad. Sci. Fenn. 243 (1957), 1-10.

[S4] K. Strebel, Zur frage der eindeutigkeit extremaler quasikonformer abbildungen des einheitskreises I and II, Comment. Math. Helv. 36 (1962), 306-323; 39 (1964), 77-89.

[S5] K. Strebel, Quadratic Differentials, Springer-Verlag, Berlin and New York, 1984.

[T] O. Teichmüller, Extremale quasikonforme abbildungen und quadratische differentiale, Abh. Preuss Akad. Wiss., Math.-Natur. Kl. 22 (1939), 1-197. 
[Y] S. Yu-Liang, On the weak convexity of $Q(R)$, Proc. Amer. Math. Soc. 124 (1996), 1879-1882.

[Z] Zhong Li, Nonuniqueness of geodesics in infinite dimensional Teichmüller spaces, Complex Variables Theory Appl. 16 (1991), 261-272.

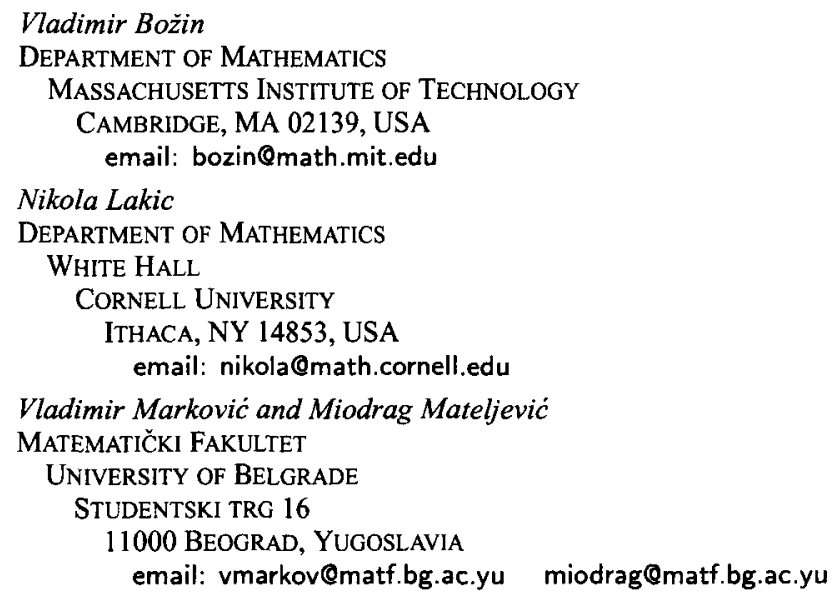

